\title{
Analysis of a stochastic predator-prey system with foraging arena scheme
}

\author{
Yongmei $\mathrm{Cai}^{1}$, Siyang $\mathrm{Cai}^{1 *}$, Xuerong $\mathrm{Mao}^{1}$ \\ ${ }^{1}$ Department of Mathematics and Statistics, University of Strathclyde, Glasgow G1 1XH, UK
}

\begin{abstract}
This paper focuses on a predator-prey system with foraging arena scheme incorporating stochastic noises. This SDE model is generated from a deterministic framework by the stochastic parameter perturbation. We then study how the correlations of the environmental noises affect the long-time behaviours of the SDE model. Later on the existence of a stationary distribution is pointed out under certain parametric restrictions. Numerical simulations are carried out to substantiate the analytical results.
\end{abstract}

Keywords: stochastic predator-prey model, Brownian motion, asymptotic pathwise estimation, stationary distribution

\section{Introduction}

A population model clarifies the mathematical relationships among consumer strategies and ecological generalities [1]. An essential element of the population models is called the functional response, which describes the density-dependent uptake response of consumers $[2,3]$. There are different types of functional responses. For example, Holling type II response $\lambda_{2}(x)=u_{1} x /\left(u_{2}+x\right)$, Holling type III response $\lambda_{2}(x)=u_{1} x^{2} /\left(u_{2}^{2}+x^{2}\right)$ [4], the ratio-dependent response $\lambda_{2}(x, y)=u_{1} x /\left(u_{2} y+x\right)[2,5-7]$ and foraging arena response $\lambda_{2}(x, y)=u_{1} x /\left(w+u_{3} y\right)=s x /(\beta+y)[3,8,9]$, where $u_{1}$ is a maximum uptake rate by the predator and $u_{2}$ is a prey half-saturation coefficient, $\beta=w / u_{3}=$ consumer density at half maximum per capita uptake rate and $u_{1} / w=s / \beta=$ maximum per capita uptake rate by predator. The two-dimensional foraging arena predator-prey model is in a form

$$
\begin{aligned}
& d \bar{x}_{1}(t)=\bar{x}_{1}(t)\left(a-b \bar{x}_{1}(t)-\frac{s \bar{x}_{2}(t)}{\beta+\bar{x}_{2}(t)}\right) d t, \\
& d \bar{x}_{2}(t)=\bar{x}_{2}(t)\left(\frac{h \bar{x}_{1}(t)}{\beta+\bar{x}_{2}(t)}-c-f \bar{x}_{2}(t)\right) d t,
\end{aligned}
$$

where $\bar{x}_{1}(t)$ and $\bar{x}_{2}(t)$ represent the population densities of prey and predator in model (1.1) at time $t$ and $a, b, s, \beta, h, c$ and $f$ are all positive constants. More precisely, $a$ is the intrinsic growth rate of prey, $c$ is the density-dependent mortality rate of consumer, $h=\phi s, b$ and $f$ are the quadratic mortality rates of prey and predator respectively. We set $\bar{x}(t)=\left(\bar{x}_{1}(t), \bar{x}_{2}(t)\right)^{T}$ as the solution of model (1.1) with the initial value $\bar{x}_{0}=\left(\bar{x}_{1}(0), \bar{x}_{2}(0)\right)^{T}$. In model (1.1), there are two non-negative trivial equilibrium points $\bar{E}_{0}=(0,0)$ and $\bar{E}_{1}=\left(\frac{a}{b}, 0\right)$. Also an unique interior equilibrium point $\bar{E}^{*}\left(\bar{x}_{1}^{*}, \bar{x}_{2}^{*}\right)$ with the nullclines

$$
\begin{aligned}
& \left(a-b \bar{x}_{1}^{*}\right)\left(\beta+\bar{x}_{2}^{*}\right)=s \bar{x}_{2}^{*}, \\
& \left(\beta+\bar{x}_{2}^{*}\right)\left(c+f \bar{x}_{2}^{*}\right)=h \bar{x}_{1}^{*}
\end{aligned}
$$

\footnotetext{
${ }^{*}$ Corresponding author and e-mail: siyang.cai@strath.ac.uk
} 
exists and is globally asymptotically stable provided that $a>\frac{b \beta c}{h}[10]$.

The deterministic models have been widely applied to explain and predict the population dynamics (e.g.[11-13]), as well as for population management and conservation (e.g.[14-20]). However, such models have their limitations in dealing with biological populations in the real world. As a result an increasing number of researchers have been studying the stochastic population systems. Mao [21] found a surprising fact that the presence of even a tiny amount of environmental noise can suppress a potential population explosion in a classical n-dimensional Lotka-Volterra model. Mao [22] compared three types of delay Lotka-Volterra models and revealed their unique properties individually. Moreover, in [23] the conditions for the SDE Lotka-Volterra model having a stationary distribution were explored and a useful method was introduced to compute the mean and variance of the stationary distribution. Further studies on the n-dimensional Lotka-Volterra models can be found in e.g.[24, 25]. Holling type II model was also well studied by many authors, e.g.[26-29]. Especially, Ji et al.[26] considered a stochastic predator-prey model with modified Leslie-Gower and Holling type II schemes and produced conditions for the system to be either extinctive or persistent. [30] studied a three-dimensional stochastic ratio-dependent model with Markov switching and Holling type III functional response. The stochastic permanence was proved mainly using the M-matrix analysis introduced by [31] and applied in [24, 25]. In [32], the asymptotic behaviours of the predator-prey system with Beddington-DeAngelis response were investigated and the conditions of having a stationary distribution were produced. However to the best of our knowledge, there has not been much work about the foraging arena model incorporating the environmental variabilities. Throughout this paper, unless otherwise specified, we let $\left(\Omega,\left\{\mathcal{F}_{t}\right\}_{t \geqslant 0}, \mathbb{P}\right)$ be a complete probability space with a filtration $\left\{\mathcal{F}_{t}\right\}$ satisfying the usual conditions (i.e.it is right continuous and increasing while $\left\{\mathcal{F}_{0}\right\}$ contains all $\mathbb{P}$-null sets). Let $B(t)=\left(B_{1}(t), \cdots, B_{6}(t)\right)^{T}$ and $\breve{B}(t)=\left(B_{1}(t), \cdots, B_{4}(t)\right)^{T}$ be six-dimensional and four-dimensional Brownian motions defined on this probability space respectively. The SDE models are then formulated as follows: Due to the environmental changes such as temperature and rainfall, we may stochastically perturb the parameters $a, c, s$ and $h$ in model (1.1) with

$$
\begin{gathered}
a \rightarrow a+\sigma_{1} \dot{B}_{1}(t), \quad c \rightarrow c+\sigma_{2} \dot{B}_{2}(t), \\
s \rightarrow s+r_{1} \dot{B}_{3}(t) \quad \text { and } \quad h \rightarrow h+r_{2} \dot{B}_{4}(t),
\end{gathered}
$$

where $\sigma_{1}, \sigma_{2}, r_{1}$ and $r_{2}$ denote the intensities of the corresponding white noise. As a result the perturbed system is given by

$$
\begin{aligned}
& d x_{1}(t)=x_{1}(t)\left(a-b x_{1}(t)-\frac{s x_{2}(t)}{\beta+x_{2}(t)}\right) d t+\sigma_{1} x_{1}(t) d B_{1}(t)-\frac{r_{1} x_{1}(t) x_{2}(t)}{\beta+x_{2}(t)} d B_{3}(t) \\
& d x_{2}(t)=x_{2}(t)\left(\frac{h x_{1}(t)}{\beta+x_{2}(t)}-c-f x_{2}(t)\right) d t-\sigma_{2} x_{2}(t) d B_{2}(t)+\frac{r_{2} x_{1}(t) x_{2}(t)}{\beta+x_{2}(t)} d B_{4}(t) .
\end{aligned}
$$

Furthermore, we would also like to incorporate perturbation into $b$ and $f$ :

$$
b \rightarrow b+\delta_{1} \dot{B}_{5}(t) \text { and } f \rightarrow f+\delta_{2} \dot{B}_{6}(t),
$$

where $\delta_{1}$ and $\delta_{2}$ represent the intensities of the corresponding white noise. We then obtain

$$
\begin{aligned}
& d x_{1}(t)=x_{1}(t)\left(a-b x_{1}(t)-\frac{s x_{2}(t)}{\beta+x_{2}(t)}\right) d t+\sigma_{1} x_{1}(t) d B_{1}(t)-\frac{r_{1} x_{1}(t) x_{2}(t)}{\beta+x_{2}(t)} d B_{3}(t)-\delta_{1} x_{1}^{2}(t) d B_{5}(t) \\
& d x_{2}(t)=x_{2}(t)\left(\frac{h x_{1}(t)}{\beta+x_{2}(t)}-c-f x_{2}(t)\right) d t-\sigma_{2} x_{2}(t) d B_{2}(t)+\frac{r_{2} x_{1}(t) x_{2}(t)}{\beta+x_{2}(t)} d B_{4}(t)-\delta_{2} x_{2}^{2}(t) d B_{6}(t) .
\end{aligned}
$$

In ecology, the spatial synchrony often occurs in the population dynamics, resulting from a synchronous random environmental factors including temperature, rainfall and sunlight etc.[33-35]. Moran effect, known as the synchronizing effect of environmental stochasticity, has been observed in multiple population models. By taking Moran effect into account, the prey and predator populations in our systems (1.2) and (1.3) can be influenced by the same external factors [35]. This phenomena can be 
characterised by the correlations between the Brownian motions affecting different species (see e.g. [35-38]). On the other hand, some environmental factors such as a disease, temperature and pollution might simultaneously affect several system parameters of a species. Therefore the correlations between the Brownian motions affecting a specific population group are also considered. As a result, we let $B(t)=\varpi Z(t)$, where $Z(t)=\left(Z_{1}(t), \cdots, Z_{6}(t)\right)^{T}$ is a six-dimensional independent standard Brownian motion and $\varpi^{T} \varpi=\mathcal{R}=\left(\rho_{i j}\right)_{6 \times 6}$ is a constant correlation matrix with $\rho_{i j} \in[-1,1]$ represents the correlation coefficient between $B_{i}(t)$ and $B_{j}(t)$ for $i, j=1,2, \cdots, 6$. And $B(t)$ can be defined in the same way. We also denote

$$
\bar{\rho}_{i j}=\left\{\begin{array}{ll}
\rho_{i j}, & \text { if } \rho_{i j}>0 \\
0, & \text { otherwise }
\end{array} \text { and } \quad \tilde{\rho}_{i j}= \begin{cases}0, & \text { if } \rho_{i j}>0 \\
-\rho_{i j}, & \text { otherwise }\end{cases}\right.
$$

We set $x(t)=\left(x_{1}(t), x_{2}(t)\right)^{T}$ as the solution of model (1.2) or model (1.3) representing the population densities of prey and predator at time $t$ with the initial value $x_{0}=\left(x_{1}(0), x_{2}(0)\right)^{T}$. Let $\mathbb{R}_{+}^{2}$ be the positive cone in $\mathbb{R}^{2}$, that is $\mathbb{R}_{+}^{2}=\left\{x \in \mathbb{R}^{2}: x_{1}>0\right.$ and $\left.x_{2}>0\right\}$. We also set $\inf \emptyset=\infty$. If $\mathrm{A}$ is a vector or matrix, its transpose is denoted by $A^{T}$. If $\mathrm{A}$ is a matrix, its trace norm is $|A|=\sqrt{\operatorname{trace}\left(A^{T} A\right)}$ whilst its operator norm is $\|A\|=\sup \{|A x|:|x|=1\}$. If $\mathrm{A}$ is a symmetric matrix, its smallest and largest eigenvalue are denoted by $\lambda_{\min }(A)$ and $\lambda_{\max }(A)$. Consider the $n$-dimensional stochastic differential equation

$$
d z(t)=\bar{f}(t) d t+\bar{g}(t) d w(t)
$$

for $t \geqslant 0$, where $z(t)=\left(z_{1}(t), \cdots, z_{n}(t)\right)^{T}$ and $w(t)=\left(w_{1}(t), \cdots, w_{n}(t)\right)^{T}$ be an $n$-dimensional Brownian motion defined on the complete probability space $\left(\Omega,\left\{\mathcal{F}_{t}\right\}_{t \geqslant 0}, \mathbb{P}\right)$ adapted to the filtration $\left\{\mathcal{F}_{t}\right\}_{t \geqslant 0}$. Let $C^{2,1}\left(\mathbb{R}^{n} \times \mathbb{R}_{+} ; \mathbb{R}\right)$ be the family of all real-valued functions $V(z, t)$ defined on $\mathbb{R}^{n} \times \mathbb{R}_{+}$ such that they are continuously twice differentiable in $z$ and once in $t$. Given $V \in C^{2,1}\left(\mathbb{R}^{n} \times \mathbb{R}_{+} ; \mathbb{R}\right)$, define an operator $L V: \mathbb{R}^{n} \times \mathbb{R}_{+} \rightarrow \mathbb{R}$ by

$$
L V(z, t)=V_{t}(z, t)+V_{z}(z, t) \bar{f}(t)+\frac{1}{2} \operatorname{trace}\left(\bar{g}^{T}(t) V_{z z}(z, t) \bar{g}(t)\right),
$$

which is called the diffusion operator of the Itô process (1.4) associated with the $C^{2,1}$-function $V$ (see e.g. [31, p. 41]). With the diffusion operator, the Itô formula (1.4) can be written as

$$
d V(z(t), t)=L V(z(t), t) d t+V_{z}(z(t), t) \bar{g}(t) d w(t) \quad \text { a.s. }
$$

This paper is divided into four main parts. In the first two parts, the unique properties of model (1.2) and (1.3) are discussed respectively including the existence and uniqueness of the positive global solution, asymptotic moment estimate and some long-time behaviours of the two species. In the third part, the parametric restrictions for either model (1.2) or (1.3) to have a stationary distribution are studied. In the final part, computer simulations based on the Euler-Maruyama scheme are performed to illustrate our theory

\section{$2 \quad$ Model (1.2)}

\subsection{Global positive solution}

Theorem 2.1. For any given initial value $x_{0} \in \mathbb{R}_{+}^{2}$, there is a unique solution $x(t)$ to equation (1.2) on $t \geqslant 0$ and the solution will remain in $\mathbb{R}_{+}^{2}$ with probability 1 , namely $x(t) \in \mathbb{R}_{+}^{2}$ for all $t \geqslant 0$ almost surely.

By defining $V(x)=x_{1}^{2}-2 \log x_{1}+x_{2}^{2}-2 \log x_{2}$, this theorem is then proved in the same routine as in $[21,39]$. 


\subsection{Asymptotic moment estimate}

Theorem 2.2. For any $\theta>0$, there exists a positive constant $K(\theta)$ such that for any initial value $x_{0} \in \mathbb{R}_{+}^{2}$, the solution of model (1.2) has the property that

$$
\limsup _{t \rightarrow \infty} \mathbb{E}|x(t)|^{\theta} \leqslant K(\theta) .
$$

Proof. Applying the Itô formula to $e^{t}\left(x_{1}^{\theta}(t)+x_{2}^{\theta}(t)\right)$ for $\theta>0$,

$$
\begin{aligned}
& e^{t}\left(x_{1}^{\theta}(t)+x_{2}^{\theta}(t)\right) \\
= & x_{1}^{\theta}(0)+x_{2}^{\theta}(0)+\int_{0}^{t} e^{s} f(x(s)) d s+\theta \sigma_{1} \int_{0}^{t} e^{s} x_{1}^{\theta}(s) d B_{1}(s)-\theta \sigma_{2} \int_{0}^{t} e^{s} x_{2}^{\theta}(s) d B_{2}(s) \\
- & \theta r_{1} \int_{0}^{t} \frac{e^{s} x_{1}^{\theta}(s) x_{2}(s)}{\beta+x_{2}(s)} d B_{3}(s)+\theta r_{2} \int_{0}^{t} \frac{e^{s} x_{2}^{\theta}(s) x_{1}(s)}{\beta+x_{2}(s)} d B_{4}(s),
\end{aligned}
$$

where

$$
\begin{aligned}
f(x) & =\theta x_{1}^{\theta}\left(a-b x_{1}-\frac{s x_{2}}{\beta+x_{2}}\right)+\theta x_{2}^{\theta}\left(\frac{h x_{1}}{\beta+x_{2}}-c-f x_{2}\right)+\frac{1}{2} \theta(\theta-1) x_{1}^{\theta}\left(\sigma_{1}^{2}+\frac{r_{1}^{2} x_{2}^{2}}{\left(\beta+x_{2}\right)^{2}}\right. \\
& \left.-\frac{2 \sigma_{1} r_{1} \rho_{13} x_{2}}{\beta+x_{2}}\right)+\frac{1}{2} \theta(\theta-1) x_{2}^{\theta}\left(\sigma_{2}^{2}+\frac{r_{2}^{2} x_{1}^{2}}{\left(\beta+x_{2}\right)^{2}}-\frac{2 r_{2} \sigma_{2} \rho_{24} x_{1}}{\beta+x_{2}}\right)+x_{1}^{\theta}+x_{2}^{\theta} .
\end{aligned}
$$

Using the elementary inequality

$$
v_{1}^{\kappa} v_{2}^{1-\kappa} \leqslant \kappa v_{1}+(1-\kappa) v_{2} \quad \text { for } v_{1}, v_{2} \geqslant 0 \text { and } 0 \leqslant \kappa<1,
$$

for $\theta \geqslant 2$ we obtain

$$
\frac{x_{1} x_{2}^{\theta}}{\beta+x_{2}} \leqslant x_{1} x_{2}^{\theta-1} \leqslant \frac{1}{\theta} x_{1}^{\theta}+\frac{\theta-1}{\theta} x_{2}^{\theta}
$$

and

$$
\frac{x_{1}^{2} x_{2}^{\theta}}{\left(\beta+x_{2}\right)^{2}} \leqslant x_{1}^{2} x_{2}^{\theta-2} \leqslant \frac{2}{\theta} x_{1}^{\theta}+\frac{\theta-2}{\theta} x_{2}^{\theta} .
$$

Hence

$$
\begin{aligned}
f(x) & \leqslant\left(h+1+a \theta+(\theta-1)\left(\frac{1}{2} \theta\left(\sigma_{1}^{2}+r_{1}^{2}+2 \sigma_{1} r_{1} \tilde{\rho}_{13}\right)+r_{2}^{2}+r_{2} \sigma_{2} \tilde{\rho}_{24}\right)\right) x_{1}^{\theta}+(1-c \theta \\
& \left.+(\theta-1)\left(h+\frac{1}{2} \theta \sigma_{2}^{2}+\frac{1}{2}(\theta-2) r_{2}^{2}+(\theta-1) r_{2} \sigma_{2} \tilde{\rho}_{24}\right)\right) x_{2}^{\theta}-b \theta x_{1}^{\theta+1}-f \theta x_{2}^{\theta+1} .
\end{aligned}
$$

is bounded, say by $K^{*}(\theta)$. Moreover, it follows from (2.1) that

$$
\mathbb{E}\left[e^{t \wedge \tau_{k}}\left(x_{1}^{\theta}\left(t \wedge \tau_{k}\right)+x_{2}^{\theta}\left(t \wedge \tau_{k}\right)\right)\right] \leqslant x_{1}^{\theta}(0)+x_{2}^{\theta}(0)+K^{*}(\theta) \int_{0}^{t \wedge \tau_{k}} e^{s} d s .
$$

Letting $k \rightarrow \infty$ and then $t \rightarrow \infty$ yields

$$
\limsup _{t \rightarrow \infty} \mathbb{E}\left[x_{1}^{\theta}(t)+x_{2}^{\theta}(t)\right] \leqslant \lim _{t \rightarrow \infty} \frac{1}{e^{t}}\left(x_{1}^{\theta}(0)+x_{2}^{\theta}(0)+K^{*}(\theta)\left(e^{t}-1\right)\right)=K^{*}(\theta) .
$$

On the other hand, we have

$$
|x|^{2} \leqslant 2\left(x_{1}^{2} \vee x_{2}^{2}\right), \text { so }|x|^{\theta} \leqslant 2^{\theta / 2}\left(x_{1}^{\theta} \vee x_{2}^{\theta}\right) \leqslant 2^{\theta / 2}\left(x_{1}^{\theta}+x_{2}^{\theta}\right) .
$$

As a result,

$$
\limsup _{t \rightarrow \infty} \mathbb{E}|x(t)|^{\theta} \leqslant 2^{\theta / 2} \limsup _{t \rightarrow \infty} \mathbb{E}\left[x_{1}^{\theta}(t)+x_{2}^{\theta}(t)\right] \leqslant 2^{\theta / 2} K^{*}(\theta)=K(\theta) .
$$

For $0<\theta<2$, Hölder's inequality yields

$$
\mathbb{E}|x(t)|^{\theta} \leqslant\left(\mathbb{E}|x(t)|^{2}\right)^{\frac{\theta}{2}} .
$$

Hence from (2.2)

$$
\limsup _{t \rightarrow \infty} \mathbb{E}|x(t)|^{\theta} \leqslant \limsup _{t \rightarrow \infty}\left(\mathbb{E}|x(t)|^{2}\right)^{\frac{\theta}{2}} \leqslant K(\theta)
$$




\subsection{Asymptotic pathwise estimation}

In order to study the asymptotic properties of model (1.2), we first introduce a lemma.

Lemma 2.3. For any initial value $x_{0} \in \mathbb{R}_{+}^{2}$, the solution of model (1.2) has the property that

$$
\limsup _{t \rightarrow \infty} \frac{1}{t} \int_{0}^{t} x_{1}^{2}(u) d u \leqslant \frac{4 a^{2}}{b^{2}} \quad \text { a.s. }
$$

Proof. According to (1.2a),

$$
x_{1}(t)=x_{1}(0)+a \int_{0}^{t} x_{1}(u) d u-b \int_{0}^{t} x_{1}^{2}(u) d u-s \int_{0}^{t} \frac{x_{1}(u) x_{2}(u)}{\beta+x_{2}(u)} d u+m_{1}(t)+m_{3}(t)
$$

where

$$
m_{1}(t)=\sigma_{1} \int_{0}^{t} x_{1}(u) d B_{1}(u) \quad \text { and } \quad m_{3}(t)=-r_{1} \int_{0}^{t} \frac{x_{1}(u) x_{2}(u)}{\beta+x_{2}(u)} d B_{3}(u)
$$

are two continuous local martingales with the quadratic variations

$$
\left\langle m_{1}(t)\right\rangle=\sigma_{1}^{2} \int_{0}^{t} x_{1}^{2}(u) d u \quad \text { and } \quad\left\langle m_{3}(t)\right\rangle=r_{1}^{2} \int_{0}^{t} \frac{x_{1}^{2}(u) x_{2}^{2}(u)}{\left(\beta+x_{2}(u)\right)^{2}} d u \leqslant r_{1}^{2} \int_{0}^{t} x_{1}^{2}(u) d u .
$$

By the exponential martingle inequality, we have

$$
\mathbb{P}\left(\sup _{o \leqslant t \leqslant n}\left(m_{i}(t)-0.5 \alpha\left\langle m_{i}(t)\right\rangle\right)>\frac{2 \log n}{\alpha}\right) \leqslant \frac{1}{n^{2}} \quad \text { for } i=1,3 \text { and } n=1,2, \cdots,
$$

where

$$
\alpha=\frac{b}{\sigma_{1}^{2}+r_{1}^{2}} .
$$

An application of the Borel-Cantelli lemma suggests that for almost all $\omega \in \Omega$ there is a random integer $n_{0}=n_{0}(\omega) \geqslant 1$ such that

$$
\sup _{o \leqslant t \leqslant n}\left(m_{i}(t)-0.5 \alpha\left\langle m_{i}(t)\right\rangle\right) \leqslant \frac{2 \log n}{\alpha} \text { whenever } n \geqslant n_{0} \text { for } i=1,3 .
$$

Hence for $t \in[0, n]$ and $n \geqslant n_{0}$,

$$
m_{i}(t) \leqslant \frac{2 \log n}{\alpha}+0.5 \alpha\left\langle m_{i}(t)\right\rangle \quad \text { a.s. }
$$

And then (2.3) and (2.4) imply that for $t \in[0, n]$ and $n \geqslant n_{0}$,

$$
\begin{aligned}
x_{1}(t) & \leqslant x_{1}(0)+a \int_{0}^{t} x_{1}(u) d u-\left(b-0.5 \alpha\left(\sigma_{1}^{2}+r_{1}^{2}\right)\right) \int_{0}^{t} x_{1}^{2}(u) d u+\frac{4 \log n}{\alpha} \\
& =x_{1}(0)+a \int_{0}^{t} x_{1}(u) d u-\frac{b}{2} \int_{0}^{t} x_{1}^{2}(u) d u+\frac{4 \log n}{\alpha} \quad \text { a.s. }
\end{aligned}
$$

Therefore it follows that for $t \in[0, n]$ and $n \geqslant n_{0}$,

$$
\begin{aligned}
\frac{b}{4} \int_{0}^{t} x_{1}^{2}(u) d u & \leqslant x_{1}(0)+a \int_{0}^{t} x_{1}(u) d u-\frac{b}{4} \int_{0}^{t} x_{1}^{2}(u) d u+\frac{4 \log n}{\alpha} \\
& \leqslant x_{1}(0)+\frac{a^{2} t}{b}+\frac{4 \log n}{\alpha} \text { a.s. }
\end{aligned}
$$

Consequently, for almost all $\omega \in \Omega$, if $n \geqslant n_{0}$ and $n-1 \leqslant t \leqslant n$,

$$
\frac{1}{t} \int_{0}^{t} x_{1}^{2}(u) d u \leqslant \frac{4}{(n-1) b}\left(x_{1}(0)+\frac{a^{2} n}{b}+\frac{4 \log n}{\alpha}\right) .
$$

Letting $t \rightarrow \infty$ and hence $n \rightarrow \infty$ we obtain

$$
\limsup _{t \rightarrow \infty} \frac{1}{t} \int_{0}^{t} x_{1}^{2}(u) d u \leqslant \frac{4 a^{2}}{b^{2}} \quad \text { a.s. }
$$


Theorem 2.4. For any initial value $x_{0} \in \mathbb{R}_{+}^{2}$,

(a) if

$$
2 a<\sigma_{1}^{2}-2 r_{1} \sigma_{1} \bar{\rho}_{13}
$$

then both $x_{1}(t)$ and $x_{2}(t)$ of model (1.2) tend to zero exponentially as $t \rightarrow \infty$ with probability 1;

(b) if

$$
\sigma_{1}^{2}+2 r_{1} \sigma_{1} \tilde{\rho}_{13}<2 a<\sigma_{1}^{2}-2 r_{1} \sigma_{1} \bar{\rho}_{13}+\frac{2 b \beta c}{h+r_{2} \sigma_{2} \rho_{24}}+\frac{b \beta \sigma_{2}^{2}}{h+r_{2} \sigma_{2} \rho_{24}} \quad \text { for } \rho_{24}>-\frac{h}{r_{2} \sigma_{2}}
$$

or

$$
2 a>\sigma_{1}^{2}+2 r_{1} \sigma_{1} \tilde{\rho}_{13} \quad \text { for }-1 \leqslant \rho_{24} \leqslant-\frac{h}{r_{2} \sigma_{2}},
$$

then $x_{1}(t)$ of model (1.2) obeys

$$
\frac{2 a-\sigma_{1}^{2}}{2 b} \leqslant \liminf _{t \rightarrow \infty} \frac{1}{t} \int_{0}^{t} x_{1}(u) d u \leqslant \limsup _{t \rightarrow \infty} \frac{1}{t} \int_{0}^{t} x_{1}(u) d u \leqslant \frac{2 a-\sigma_{1}^{2}+2 r_{1} \sigma_{1} \bar{\rho}_{13}}{2 b} \quad \text { a.s. }
$$

and $x_{2}(t)$ tends to zero exponentially as $t \rightarrow \infty$ with probability 1.

Proof. (a) Applying Itô's formula on $\log x_{1}$ yields

$$
\begin{aligned}
d \log x_{1}(t) & =\left(a-b x_{1}(t)-\frac{1}{2} \sigma_{1}^{2}-\frac{s x_{2}(t)}{\beta+x_{2}(t)}-\frac{r_{1}^{2} x_{2}^{2}(t)}{2\left(\beta+x_{2}(t)\right)^{2}}+\frac{r_{1} \sigma_{1} \rho_{13} x_{2}(t)}{\beta+x_{2}(t)}\right) d t+\sigma_{1} d B_{1}(t) \\
& -\frac{r_{1} x_{2}(t)}{\beta+x_{2}(t)} d B_{3}(t) \\
& \leqslant\left(a-\frac{1}{2} \sigma_{1}^{2}+r_{1} \sigma_{1} \bar{\rho}_{13}\right) d t+\sigma_{1} d B_{1}(t)-\frac{r_{1} x_{2}(t)}{\beta+x_{2}(t)} d B_{3}(t) .
\end{aligned}
$$

Integrating from 0 to $t$ and dividing by $t$ infers

$$
\frac{1}{t} \log x_{1}(t) \leqslant \frac{1}{t} \log x_{1}(0)+a-\frac{1}{2} \sigma_{1}^{2}+r_{1} \sigma_{1} \bar{\rho}_{13}+\frac{M_{1}(t)}{t}+\frac{M_{3}(t)}{t},
$$

where

$$
M_{1}(t)=\sigma_{1} B_{1}(t) \quad \text { and } \quad M_{3}(t)=-r_{1} \int_{0}^{t} \frac{x_{2}(u)}{\beta+x_{2}(u)} d B_{3}(u)
$$

are two continuous martingales with the quadratic variations

$$
\left\langle M_{1}(t)\right\rangle=\sigma_{1}^{2} t \quad \text { and } \quad\left\langle M_{3}(t)\right\rangle=r_{1}^{2} \int_{0}^{t} \frac{x_{2}^{2}(u)}{\left(\beta+x_{2}(u)\right)^{2}} d t \leqslant r_{1}^{2} t .
$$

By the strong law of large numbers for martingales [31, 40],

$$
\lim _{t \rightarrow \infty} \frac{M_{1}(t)}{t}=0 \quad \text { and } \quad \lim _{t \rightarrow \infty} \frac{M_{3}(t)}{t}=0 \quad \text { a.s. }
$$

and thus from condition $(2.5)$

$$
\limsup _{t \rightarrow \infty} \frac{1}{t} \log x_{1}(t) \leqslant a-\frac{1}{2} \sigma_{1}^{2}+r_{1} \sigma_{1} \bar{\rho}_{13}<0 \quad \text { a.s. }
$$

as required. Therefore we obtain

$$
\lim _{t \rightarrow \infty} \frac{1}{t} \int_{0}^{t} x_{1}(u) d u=0 \quad \text { a.s. }
$$


Meanwhile

$$
\begin{aligned}
d \log x_{2}(t) & =\left(\frac{h+r_{2} \sigma_{2} \rho_{24}}{\beta+x_{2}(t)} x_{1}(t)-c-\frac{\sigma_{2}^{2}}{2}-f x_{2}(t)-\frac{r_{2}^{2} x_{1}^{2}(t)}{2\left(\beta+x_{2}(t)\right)^{2}}\right) d t-\sigma_{2} d B_{2}(t) \\
& +\frac{r_{2} x_{1}(t)}{\beta+x_{2}(t)} d B_{4}(t) .
\end{aligned}
$$

It follows that

$$
\frac{\log x_{2}(t)}{t} \leqslant \frac{1}{t}\left(\log x_{2}(0)+\frac{h+r_{2} \sigma_{2} \bar{\rho}_{24}}{\beta} \int_{0}^{t} x_{1}(u) d u\right)-\left(c+\frac{\sigma_{2}^{2}}{2}\right)+\frac{M_{2}(t)}{t}+\frac{M_{4}(t)}{t},
$$

where

$$
M_{2}(t)=-\sigma_{2} B_{2}(t) \quad \text { and } \quad M_{4}(t)=r_{2} \int_{0}^{t} \frac{x_{1}(u)}{\beta+x_{2}(u)} d B_{4}(u)
$$

are two martingales with the quadratic variations

$$
\left\langle M_{2}(t)\right\rangle=\sigma_{2}^{2} t \quad \text { and } \quad\left\langle M_{4}(t)\right\rangle=r_{2}^{2} \int_{0}^{t} \frac{x_{1}^{2}(u)}{\left(\beta+x_{2}(u)\right)^{2}} d u .
$$

Hence from Lemma 2.3,

$$
\limsup _{t \rightarrow \infty} \frac{\left\langle M_{4}(t)\right\rangle}{t} \leqslant \limsup _{t \rightarrow \infty} \frac{r_{2}^{2}}{\beta^{2} t} \int_{0}^{t} x_{1}^{2}(u) d u \leqslant \frac{4 r_{2}^{2} a^{2}}{\beta^{2} b^{2}} \quad \text { a.s. }
$$

By the strong law of large numbers for martingales,

$$
\lim _{t \rightarrow \infty} \frac{M_{2}(t)}{t}=0 \quad \text { and } \quad \lim _{t \rightarrow \infty} \frac{M_{4}(t)}{t}=0 \quad \text { a.s. }
$$

Letting $t \rightarrow \infty$ and recalling equation (2.9) indicates

$$
\limsup _{t \rightarrow \infty} \frac{\log x_{2}(t)}{t} \leqslant-\left(c+\frac{\sigma_{2}^{2}}{2}\right)<0 \quad \text { a.s. }
$$

(b) Applying Itô's formula on $\frac{1}{x_{1}(t)}$ gives

$$
\begin{aligned}
d\left(\frac{1}{x_{1}(t)}\right) & =\left(\frac{1}{x_{1}}\left(\frac{s x_{2}}{\beta+x_{2}}-a+\sigma_{1}^{2}+\frac{r_{1}^{2} x_{2}^{2}}{\left(\beta+x_{2}\right)^{2}}-\frac{2 \sigma_{1} r_{1} \rho_{13} x_{2}}{\beta+x_{2}}\right)+b\right) d t-\frac{\sigma_{1}}{x_{1}} d B_{1}(t) \\
& +\frac{r_{1} x_{2}}{x_{1}\left(\beta+x_{2}\right)} d B_{3}(t),
\end{aligned}
$$

where we write $x(t)=x$. Hence by the variation-of-constants formula (see e.g. [40, pp. 98-99]),

$$
\begin{aligned}
\frac{1}{x_{1}(t)} & =\exp \left(\int_{0}^{t}\left(\frac{1}{2} \sigma_{1}^{2}-a+\frac{s x_{2}(u)}{\beta+x_{2}(u)}+\frac{r_{1}^{2} x_{2}^{2}(u)}{2\left(\beta+x_{2}(u)\right)^{2}}-\frac{2 r_{1} \sigma_{1} \rho_{13} x_{2}(u)}{\beta+x_{2}(u)}\right) d u-M_{1}(t)\right. \\
& \left.-M_{3}(t)\right)\left(\frac{1}{x_{1}(0)}+b \int_{0}^{t} \exp \left(\int _ { 0 } ^ { u } \left(a-\frac{s x_{2}(v)}{\beta+x_{2}(v)}-\frac{1}{2} \sigma_{1}^{2}-\frac{r_{1}^{2} x_{2}^{2}(v)}{2\left(\beta+x_{2}(v)\right)^{2}}\right.\right.\right. \\
& \left.\left.\left.+\frac{2 r_{1} \sigma_{1} \rho_{13} x_{2}(v)}{\beta+x_{2}(v)}\right) d v+M_{1}(u)+M_{3}(u)\right) d u\right) \\
& =\exp \left(-M_{1}(t)-M_{3}(t)\right)\left(\frac { 1 } { x _ { 1 } ( 0 ) } \operatorname { e x p } \left(-\left(a-\frac{1}{2} \sigma_{1}^{2}\right) t+s \int_{0}^{t} \frac{x_{2}(u)}{\beta+x_{2}(u)} d u\right.\right. \\
& \left.+\frac{r_{1}^{2}}{2} \int_{0}^{t} \frac{x_{2}^{2}(u)}{\left(\beta+x_{2}(u)\right)^{2}} d u-2 r_{1} \sigma_{1} \rho_{13} \int_{0}^{t} \frac{x_{2}(u)}{\beta+x_{2}(u)} d u\right)+b \int_{0}^{t} \exp \left(-\left(a-\frac{1}{2} \sigma_{1}^{2}\right)(t-u)\right. \\
& +s \int_{u}^{t} \frac{x_{2}(v)}{\beta+x_{2}(v)} d v+\frac{r_{1}^{2}}{2} \int_{u}^{t} \frac{x_{2}^{2}(v)}{\left(\beta+x_{2}(v)\right)^{2}} d v-2 r_{1} \sigma_{1} \rho_{13} \int_{u}^{t} \frac{x_{2}(v)}{\beta+x_{2}(v)} d v+M_{1}(u) \\
& \left.\left.+M_{3}(u)\right) d u\right) .
\end{aligned}
$$


On the one hand, (2.11) leads to

$$
\begin{aligned}
\frac{1}{x_{1}(t)} & \leqslant \exp \left(-M_{1}(t)-M_{3}(t)\right)\left(\frac { 1 } { x _ { 1 } ( 0 ) } \operatorname { e x p } \left(-\left(a-\frac{1}{2} \sigma_{1}^{2}\right) t+s \int_{0}^{t} \frac{x_{2}(u)}{\beta+x_{2}(u)} d u\right.\right. \\
& \left.+\frac{r_{1}^{2}}{2} \int_{0}^{t} \frac{x_{2}^{2}(u)}{\left(\beta+x_{2}(u)\right)^{2}} d u+2 r_{1} \sigma_{1} \tilde{\rho}_{13} \int_{0}^{t} \frac{x_{2}(u)}{\beta+x_{2}(u)} d u\right)+b \exp \left(\max _{0 \leqslant u \leqslant t} M_{1}(u)+\max _{0 \leqslant u \leqslant t} M_{3}(u)\right. \\
& \left.+s \int_{0}^{t} \frac{x_{2}(u)}{\beta+x_{2}(u)} d u+\frac{r_{1}^{2}}{2} \int_{0}^{t} \frac{x_{2}^{2}(u)}{\left(\beta+x_{2}(u)\right)^{2}} d u+r_{1} \sigma_{1} \tilde{\rho}_{13} \int_{0}^{t} \frac{x_{2}(u)}{\beta+x_{2}(u)} d u\right) . \\
& \left.\int_{0}^{t} \exp (-q(t-u)) d u\right) \\
& \leqslant \exp \left(\max _{0 \leqslant u \leqslant t} M_{1}(u)-M_{1}(t)+\max _{0 \leqslant u \leqslant t} M_{3}(u)-M_{3}(t)+s \int_{0}^{t} \frac{x_{2}(u)}{\beta+x_{2}(u)} d u\right. \\
& \left.+\frac{r_{1}^{2}}{2} \int_{0}^{t} \frac{x_{2}^{2}(u)}{\left(\beta+x_{2}(u)\right)^{2}} d u+r_{1} \sigma_{1} \tilde{\rho}_{13} \int_{0}^{t} \frac{x_{2}(u)}{\beta+x_{2}(u)} d u\right)\left(\frac{1}{x_{1}(0)} \exp (-q t)\right. \\
& \left.+b \int_{0}^{t} \exp (-q(t-u)) d u\right) \\
& =\exp \left(\max _{0 \leqslant u \leqslant t} M_{1}(u)-M_{1}(t)+\max _{0 \leqslant u \leqslant t} M_{3}(u)-M_{3}(t)+s \int_{0}^{t} \frac{x_{2}(u)}{\beta+x_{2}(u)} d u\right. \\
& \left.+\frac{r_{1}^{2}}{2} \int_{0}^{t} \frac{x_{2}^{2}(u)}{\left(\beta+x_{2}(u)\right)^{2}} d u+r_{1} \sigma_{1} \tilde{\rho}_{13} \int_{0}^{t} \frac{x_{2}(u)}{\beta+x_{2}(u)} d u\right) \cdot K_{1}(t),
\end{aligned}
$$

where

$$
q:=a-\frac{1}{2} \sigma_{1}^{2}-r_{1} \sigma_{1} \tilde{\rho}_{13} \text { and } K_{1}(t)=\frac{1}{x_{1}(0)} \exp (-q t)+\frac{2 b(1-\exp (-q t))}{2 a-\sigma_{1}^{2}} .
$$

Under condition (2.6) or (2.7), we obtain that $q>0$ and therefore $\sup _{0 \leqslant t<\infty} K_{1}(t)<\infty$. It then follows that

$$
\begin{aligned}
\frac{\log x_{1}(t)}{t} & \geqslant-\frac{\log K_{1}(t)}{t}-\frac{\max _{0 \leqslant u \leqslant t} M_{1}(u)-M_{1}(t)+\max _{0 \leqslant u \leqslant t} M_{3}(u)-M_{3}(t)}{t} \\
& -\frac{s}{t} \int_{0}^{t} \frac{x_{2}(u)}{\beta+x_{2}(u)} d u-\frac{r_{1}^{2}}{2 t} \int_{0}^{t} \frac{x_{2}^{2}(u)}{\left(\beta+x_{2}(u)\right)^{2}} d u-\frac{r_{1} \sigma_{1} \tilde{\rho}_{13}}{t} \int_{0}^{t} \frac{x_{2}(u)}{\beta+x_{2}(u)} d u .
\end{aligned}
$$

By (2.8) and (2.12),

$$
\begin{aligned}
\frac{1}{t} \int_{0}^{t} x_{1}(u) d u & =\frac{2 a-\sigma_{1}^{2}}{2 b}-\frac{\log x_{1}(t)}{b t}+\frac{\log x_{1}(0)}{b t}-\frac{s}{b t} \int_{0}^{t} \frac{x_{2}(u)}{\beta+x_{2}(u)} d u \\
& -\frac{r_{1}^{2}}{2 b t} \int_{0}^{t} \frac{x_{2}^{2}(u)}{\left(\beta+x_{2}(u)\right)^{2}} d u+\frac{r_{1} \sigma_{1} \rho_{13}}{b t} \int_{0}^{t} \frac{x_{2}(u)}{\beta+x_{2}(u)} d u+\frac{M_{1}(t)}{b t}+\frac{M_{3}(t)}{b t} \\
& \leqslant \frac{2 a-\sigma_{1}^{2}}{2 b}+\frac{\log K_{1}(t)}{b t}+\frac{\max _{0 \leqslant u \leqslant t} M_{1}(u)-M_{1}(t)+\max _{0 \leqslant u \leqslant t} M_{3}(u)-M_{3}(t)}{b t} \\
& +\frac{r_{1} \sigma_{1} \bar{\rho}_{13}}{b}+\frac{\log x_{1}(0)}{b t}+\frac{M_{1}(t)}{b t}+\frac{M_{3}(t)}{b t}
\end{aligned}
$$

As $t \rightarrow \infty$ and from the strong law of large numbers for martingales,

$$
\limsup _{t \rightarrow \infty} \frac{1}{t} \int_{0}^{t} x_{1}(u) d u \leqslant \frac{2 a-\sigma_{1}^{2}+2 r_{1} \sigma_{1} \bar{\rho}_{13}}{2 b} \text { a.s. }
$$

Assume that $\rho_{24}>-\frac{h}{r_{2} \sigma_{2}}$. From equation (2.10),

$$
d \log x_{2}(t) \leqslant\left(\frac{h+r_{2} \sigma_{2} \rho_{24}}{\beta} x_{1}(t)-c-\frac{\sigma_{2}^{2}}{2}\right) d t-\sigma_{2} d B_{2}(t)+\frac{r_{2} x_{1}(t)}{\beta+x_{2}(t)} d B_{4}(t) .
$$


It is then followed from (2.14) and the strong law of large numbers for martingales that

$$
\begin{aligned}
\limsup _{t \rightarrow \infty} \frac{1}{t} \log x_{2}(t) & \leqslant \frac{h+r_{2} \sigma_{2} \rho_{24}}{\beta} \limsup _{t \rightarrow \infty} \frac{1}{t} \int_{0}^{t} x_{1}(u) d u-\left(c+\frac{\sigma_{2}^{2}}{2}\right) \\
& \leqslant \frac{\left(h+r_{2} \sigma_{2} \rho_{24}\right)\left(2 a-\sigma_{1}^{2}+2 r_{1} \sigma_{1} \bar{\rho}_{13}\right)}{2 \beta b}-\left(c+\frac{\sigma_{2}^{2}}{2}\right)<0
\end{aligned}
$$

in view of (2.6). If $\rho_{24} \leqslant-\frac{h}{r_{2} \sigma_{2}}$, it immediately indicates that

$$
\limsup _{t \rightarrow \infty} \frac{1}{t} \log x_{2}(t) \leqslant-\left(c+\frac{\sigma_{2}^{2}}{2}\right)<0 \quad \text { a.s. }
$$

Hence for arbitrary small $\zeta>0$, there exists $t_{\zeta}(\omega)$ such that

$$
\mathbb{P}\left(\Omega_{\zeta}\right) \geqslant 1-\zeta \text { where } \Omega_{\zeta}=\left\{\omega: \frac{\left(s+r_{1} \sigma_{1}\left|\rho_{13}\right|\right) x_{2}(t, \omega)}{b\left(\beta+x_{2}(t, \omega)\right)}+\frac{r_{1}^{2} x_{2}^{2}(t, \omega)}{2 b\left(\beta+x_{2}(t, \omega)\right)^{2}} \leqslant \zeta \text { for all } t \geqslant t_{\zeta}\right\} .
$$

On the other hand, (2.11) yields

$$
\begin{aligned}
\frac{1}{x_{1}(t)} \geqslant & \exp \left(-M_{1}(t)-M_{3}(t)\right)\left(\frac{1}{x_{1}(0)} \exp \left(-\left(a-\frac{1}{2} \sigma_{1}^{2}\right) t-2 r_{1} \sigma_{1} \bar{\rho}_{13} \int_{0}^{t} \frac{x_{2}(u)}{\beta+x_{2}(u)} d u\right)\right. \\
& +b \exp \left(\min _{0 \leqslant u \leqslant t} M_{1}(u)+\min _{0 \leqslant u \leqslant t} M_{3}(u)-2 r_{1} \sigma_{1} \bar{\rho}_{13} \int_{0}^{t} \frac{x_{2}(u)}{\beta+x_{2}(u)} d u\right) . \\
& \left.\int_{0}^{t} \exp \left(-\left(a-\frac{1}{2} \sigma_{1}^{2}\right)(t-u)\right) d u\right) \\
& \geqslant \exp \left(\min _{0 \leqslant u \leqslant t} M_{1}(u)-M_{1}(t)+\min _{0 \leqslant u \leqslant t} M_{3}(u)-M_{3}(t)-2 r_{1} \sigma_{1} \bar{\rho}_{13} \int_{0}^{t} \frac{x_{2}(u)}{\beta+x_{2}(u)} d u\right) \cdot K_{2}(t),
\end{aligned}
$$

where

$$
K_{2}(t)=\frac{1}{x_{1}(0)} \exp \left(-\left(a-\frac{1}{2} \sigma_{1}^{2}\right) t\right)+\frac{2 b\left(1-\exp \left(-\left(a-\frac{1}{2} \sigma_{1}^{2}\right) t\right)\right)}{2 a-\sigma_{1}^{2}}
$$

and $\sup _{0 \leqslant t<\infty} K_{2}(t)<\infty$ if either condition (2.6) or (2.7) holds. Then

$$
\begin{aligned}
\frac{\log x_{1}(t)}{t} \leqslant & -\frac{\log K_{2}(t)}{t}-\frac{\min _{0 \leqslant u \leqslant t} M_{1}(u)-M_{1}(t)+\min _{0 \leqslant u \leqslant t} M_{3}(u)-M_{3}(t)}{t} \\
& +\frac{2 r_{1} \sigma_{1} \bar{\rho}_{13}}{t} \int_{0}^{t} \frac{x_{2}(u)}{\beta+x_{2}(u)} d u .
\end{aligned}
$$

Hence we obtain from (2.13) that

$$
\begin{aligned}
\frac{1}{t} \int_{0}^{t} x_{1}(u) d u & \geqslant \frac{2 a-\sigma_{1}^{2}}{2 b}+\frac{\log K_{2}(t)}{b t}+\frac{\min _{0 \leqslant u \leqslant t} M_{1}(u)-M_{1}(t)+\min _{0 \leqslant u \leqslant t} M_{3}(u)-M_{3}(t)}{b t} \\
& +\frac{\log x_{1}(0)}{b t}-\frac{s}{b t} \int_{0}^{t} \frac{x_{2}(u)}{\beta+x_{2}(u)} d u-\frac{r_{1}^{2}}{2 b t} \int_{0}^{t} \frac{x_{2}^{2}(u)}{\left(\beta+x_{2}(u)\right)^{2}} d u \\
& -\frac{r_{1} \sigma_{1}\left|\rho_{13}\right|}{b t} \int_{0}^{t} \frac{x_{2}(u)}{\beta+x_{2}(u)} d u+\frac{M_{1}(t)}{b t}+\frac{M_{3}(t)}{b t}
\end{aligned}
$$

For any $\omega \in \Omega_{\zeta},(2.15)$ indicates

$$
\liminf _{t \rightarrow \infty} \frac{1}{t} \int_{0}^{t} x_{1}(u) d u \geqslant \frac{2 a-\sigma_{1}^{2}}{2 b}-\zeta \text { a.s. }
$$

Letting $\zeta \rightarrow 0$ and together with (2.14) implies the required assertion.

Remark 2.5. Let all the Brownian motions in model (1.2) be uncorrelated. Then Theorem 2.2 is still obtained. Moreover, Theorem 2.4(a) or (b) holds if assertion (2.5) or (2.6) is satisfied with $\rho_{i j}=0$ for all $i, j=1, \cdots, 4$ and $i \neq j$. 
Remark 2.6. Assume that $\rho_{13} \leqslant 0$. Then under condition (2.6) or (2.7), $x_{1}(t)$ of model (1.2) obeys

$$
\lim _{t \rightarrow \infty} \frac{1}{t} \int_{0}^{t} x_{1}(u) d u=\frac{2 a-\sigma_{1}^{2}}{2 b} \text { a.s. }
$$

and $x_{2}(t)$ tends to zero exponentially as $t \rightarrow \infty$ with probability 1.

Theorem 2.4(a) shows that large white noise intensity $\sigma_{1}^{2}$ may let the populations die out. In Theorem 2.4(b), the situation where $a$ becomes larger is discussed. There are generally two cases, depending on the value of $\rho_{24}$. In the first case, $B_{2}(t)$ and $B_{4}(t)$ are strongly negatively correlated $\left(-1 \leqslant \rho_{24} \leqslant-\frac{h}{r_{2} \sigma_{2}}\right)$. Then under condition $(2.7)$, the prey species keep persistent while the consumers become extinct ultimately. On the other hand, we let $\rho_{24}>-\frac{h}{r_{2} \sigma_{2}}$. Then system (1.2) has the same behaviours as in the first case provided that (2.6) is fulfilled. It is then interesting to examine how the population system behaves when $a$ gets larger in the case $\rho_{24}>-\frac{h}{r_{2} \sigma_{2}}$. This is further developed in section 4.

\section{Model (1.3)}

In this section, we investigate the long-time behaviours of model (1.3). Notice that if $\delta_{1}$ and $\delta_{2}$ are zero, model (1.3) is then degenerated to model (1.2) which has been analysed as above. Hence this section only focuses on the unique properties of model (1.3) with two positive constants $\delta_{1}$ and $\delta_{2}$.

\subsection{Global positive solution}

Theorem 3.1. For any given initial value $x_{0} \in \mathbb{R}_{+}^{2}$, there is a unique solution $x(t)$ to equation (1.3) on $t \geqslant 0$ and the solution will remain in $\mathbb{R}_{+}^{2}$ with probability 1 , namely $x(t) \in \mathbb{R}_{+}^{2}$ for all $t \geqslant 0$ a.s.

By defining $V(x)=x_{1}^{0.5}-0.5 \log x_{1}+x_{2}^{0.5}-0.5 \log x_{2}$, this theorem is then proved in the same routine as in $[21,39]$.

\section{$3.2 \quad$ Asymptotic moment estimate}

Theorem 3.2. Let $\eta_{1}$ and $\eta_{2}$ be positive numbers satisfying

$$
\begin{aligned}
\eta_{1}, \eta_{2} & <\frac{1}{2} \text { for } \rho_{45} \geqslant 0 \text { and } \rho_{56} \leqslant 0 ; \\
\eta_{1}+2 \eta_{2} & <\frac{1}{2} \text { for } \rho_{45}<0 \text { and } \rho_{56}>0 \\
\eta_{1}+\eta_{2} & <\frac{1}{2} \quad \text { otherwise. }
\end{aligned}
$$

Then for any initial value $x_{0} \in \mathbb{R}_{+}^{2}$, the solution of model (1.3) has the property that

$$
\limsup _{t \rightarrow \infty} \mathbb{E}\left[x_{1}^{\eta_{1}}(t) x_{2}^{\eta_{2}}(t)\right] \leqslant e^{c_{1} / c_{2}}
$$

where $c_{1}$ and $c_{2}$ are two constants determined in (3.5) and (3.6) below in the case $\rho_{45}<0$ and $\rho_{56}>0$.

In order to prove this theorem, let us first consider the following lemma.

Lemma 3.3. Let $\eta_{1}$ and $\eta_{2}$ be positive numbers satisfying

$$
\begin{aligned}
\eta_{1}, \eta_{2} & <1 \text { for } \rho_{45} \geqslant 0 \text { and } \rho_{56} \leqslant 0 \\
\eta_{1}+2 \eta_{2} & <1 \text { for } \rho_{45}<0 \text { and } \rho_{56}>0 \\
\eta_{1}+\eta_{2} & <1 \text { otherwise, }
\end{aligned}
$$

Then for any initial value $x_{0} \in \mathbb{R}_{+}^{2}$, the solution of model (1.3) has the property that

$$
\mathbb{E}\left[x_{1}^{\eta_{1}}(t) x_{2}^{\eta_{2}}(t)\right]<\infty \text { for all } t \geqslant 0 .
$$


Proof. Define a $C^{2}$-function $V: \mathbb{R}_{+}^{2} \rightarrow \mathbb{R}_{+}$by $V(x)=x_{1}^{\eta_{1}} x_{2}^{\eta_{2}}$. And we obtain

$$
\begin{aligned}
L V(x) & \leqslant V(x)\left[A_{1}+A_{2} x_{1}+A_{3} x_{2}+\frac{1}{2} \delta_{1}^{2} \eta_{1}\left(\eta_{1}-1\right) x_{1}^{2}+\frac{1}{2} \delta_{2}^{2} \eta_{2}\left(\eta_{2}-1\right) x_{2}^{2}+\frac{\eta_{2}\left(\eta_{2}-1\right) r_{2}^{2}}{2\left(\beta+x_{2}\right)^{2}} x_{1}^{2}\right. \\
& \left.-\frac{\delta_{1} r_{2} \eta_{1} \eta_{2} \rho_{45}}{\beta+x_{2}} x_{1}^{2}+\delta_{1} \delta_{2} \eta_{1} \eta_{2} \rho_{56} x_{1} x_{2}\right]
\end{aligned}
$$

where

$$
\begin{gathered}
A_{1}=a \eta_{1}-c \eta_{2}-\sigma_{1} \sigma_{2} \eta_{1} \eta_{2} \rho_{12}+\sigma_{1} r_{1} \eta_{1}\left(1-\eta_{1}\right) \bar{\rho}_{13}+r_{1} \sigma_{2} \eta_{1} \eta_{2} \bar{\rho}_{23} \\
A_{2}=-b \eta_{1}+\frac{h \eta_{2}}{\beta}+\frac{\sigma_{1} r_{2} \eta_{1} \eta_{2} \bar{\rho}_{14}}{\beta}+\delta_{1} \sigma_{1} \eta_{1}\left(1-\eta_{1}\right) \rho_{15}+\frac{\sigma_{2} r_{2} \eta_{2}\left(1-\eta_{2}\right) \bar{\rho}_{24}}{\beta}+\sigma_{2} \delta_{1} \eta_{1} \eta_{2} \rho_{25} \\
+\frac{r_{1} r_{2} \eta_{1} \eta_{2} \tilde{\rho}_{34}}{\beta}+\delta_{1} r_{1} \eta_{1}\left(1-\eta_{1}\right) \tilde{\rho}_{35}+\delta_{2} r_{2} \eta_{2}\left(1-\eta_{2}\right) \bar{\rho}_{46}
\end{gathered}
$$

and

$$
A_{3}=-f \eta_{2}-\sigma_{1} \delta_{2} \eta_{2} \eta_{1} \rho_{16}-\delta_{2} \sigma_{2} \eta_{2}\left(1-\eta_{2}\right) \rho_{26}+r_{1} \delta_{2} \eta_{1} \eta_{2} \bar{\rho}_{36}
$$

Assuming that $\rho_{45}<0$ and $\rho_{56}>0$, we obtain

$$
\begin{aligned}
& \frac{1}{4} \delta_{1}^{2} \eta_{1}\left(\eta_{1}-1\right) x_{1}^{2}+\frac{\eta_{2}\left(\eta_{2}-1\right) r_{2}^{2}}{2\left(\beta+x_{2}\right)^{2}} x_{1}^{2}-\frac{\delta_{1} r_{2} \eta_{1} \eta_{2} \rho_{45}}{\beta+x_{2}} x_{1}^{2} \\
\leqslant & \frac{1}{4} \delta_{1}^{2} \eta_{1}^{2} x_{1}^{2}-\frac{1}{4} \delta_{1}^{2} \eta_{1} x_{1}^{2}+\frac{\eta_{2}^{2} r_{2}^{2} x_{1}^{2}}{2\left(\beta+x_{2}\right)^{2}}-\frac{\eta_{2} r_{2}^{2} x_{1}^{2}}{2\left(\beta+x_{2}\right)^{2}}+\frac{1}{2} \eta_{1} \eta_{2}\left(\frac{r_{2}^{2} x_{1}^{2}}{\left(\beta+x_{2}\right)^{2}}+\delta_{1}^{2} x_{1}^{2}\right) \\
= & \frac{1}{4} \eta_{1}\left(\eta_{1}+2 \eta_{2}-1\right) \delta_{1}^{2} x_{1}^{2}+\frac{1}{2} \eta_{2}\left(\eta_{1}+\eta_{2}-1\right) \frac{r_{2}^{2} x_{1}^{2}}{\left(\beta+x_{2}\right)^{2}}
\end{aligned}
$$

and

$$
\begin{aligned}
& \frac{1}{4} \delta_{1}^{2} \eta_{1}\left(\eta_{1}-1\right) x_{1}^{2}+\frac{1}{2} \delta_{2}^{2} \eta_{2}\left(\eta_{2}-1\right) x_{2}^{2}+\delta_{1} \delta_{2} \eta_{1} \eta_{2} \rho_{56} x_{1} x_{2} \\
\leqslant & \frac{1}{4} \delta_{1}^{2} \eta_{1}^{2} x_{1}^{2}-\frac{1}{4} \delta_{1}^{2} \eta_{1} x_{1}^{2}+\frac{1}{2} \delta_{2}^{2} \eta_{2}^{2} x_{2}^{2}-\frac{1}{2} \delta_{2}^{2} \eta_{2} x_{2}^{2}+\frac{1}{2} \eta_{1} \eta_{2}\left(\delta_{1}^{2} x_{1}^{2}+\delta_{2}^{2} x_{2}^{2}\right) \\
= & \frac{1}{4} \eta_{1}\left(\eta_{1}+2 \eta_{2}-1\right) \delta_{1}^{2} x_{1}^{2}+\frac{1}{2} \eta_{2}\left(\eta_{1}+\eta_{2}-1\right) \delta_{2}^{2} x_{2}^{2} .
\end{aligned}
$$

Hence

$$
L V(x) \leqslant V(x)\left(A_{1}+A_{2} x_{1}+A_{3} x_{2}-\frac{1}{2} \eta_{1}\left(1-\left(\eta_{1}+2 \eta_{2}\right)\right) \delta_{1}^{2} x_{1}^{2}-\frac{1}{2} \eta_{2}\left(1-\left(\eta_{1}+\eta_{2}\right)\right) \delta_{2}^{2} x_{2}^{2}\right) .
$$

As the polynomial

$$
A_{1}+A_{2} x_{1}+A_{3} x_{2}-\frac{1}{4} \eta_{1}\left(1-\left(\eta_{1}+2 \eta_{2}\right)\right) \delta_{1}^{2} x_{1}^{2}-\frac{1}{4} \eta_{2}\left(1-\left(\eta_{1}+\eta_{2}\right)\right) \delta_{2}^{2} x_{2}^{2}
$$

is bounded by

$$
c_{1}=\frac{\eta_{1}\left(1-\left(\eta_{1}+2 \eta_{2}\right)\right) \delta_{1}^{2} A_{1}+A_{2}^{2}}{\eta_{1}\left(1-\left(\eta_{1}+2 \eta_{2}\right)\right) \delta_{1}^{2}}+\frac{A_{3}^{2}}{\eta_{2}\left(1-\left(\eta_{1}+\eta_{2}\right)\right) \delta_{2}^{2}},
$$

we obtain

$$
L V(x) \leqslant V(x)\left(c_{1}-c_{2}|x|^{2}\right),
$$

where

$$
c_{2}=\frac{1}{4}\left(\eta_{1}\left(1-\left(\eta_{1}+2 \eta_{2}\right)\right) \delta_{1}^{2} \wedge \eta_{2}\left(1-\left(\eta_{1}+\eta_{2}\right)\right) \delta_{2}^{2}\right)
$$


This leads to

$$
\mathbb{E} V\left(x\left(t \wedge \tau_{k}\right)\right)=V\left(x_{0}\right)+\mathbb{E} \int_{0}^{t \wedge \tau_{k}} L V(x(s)) d s \leqslant V\left(x_{0}\right)+c_{1} \int_{0}^{t} \mathbb{E} V\left(x\left(s \wedge \tau_{k}\right)\right) d s .
$$

It then follows from the Gronwall inequality that

$$
\mathbb{E} V\left(x\left(t \wedge \tau_{k}\right)\right) \leqslant V\left(x_{0}\right) e^{c_{1} t} .
$$

Letting $k \rightarrow \infty$ implies

$$
\mathbb{E} V(x(t)) \leqslant V\left(x_{0}\right) e^{c_{1} t}<\infty \text { for all } t \geqslant 0 .
$$

Similarly, one can deduce the same results under condition (3.1a) or (3.1c) with the corresponding values of $c_{1}$ and $c_{2}$. Here it is omitted.

Proof of Theorem 3.2. This proof is standard by using the results of Lemma 3.3. One can refer to [21, pp. 104-105] for details.

We can obtain from the Chebyshev's inequality and Theorem 3.2 that

$$
\begin{aligned}
& \mathbb{P}\left(x_{1} \geqslant D_{1} \text { and } x_{2} \geqslant D_{2}\right) \\
= & \mathbb{E}\left[\mathbf{I}_{\left\{x_{1} \geqslant D_{1}\right\}} \mathbf{I}_{\left\{x_{2} \geqslant D_{2}\right\}}\right] \leqslant \mathbb{E}\left[\frac{x_{1}^{\eta_{1}}}{D_{1}^{\eta_{1}}} \mathbf{I}_{\left\{x_{1} \geqslant D_{1}\right\}} \frac{x_{2}^{\eta_{2}}}{D_{2}^{\eta_{2}}} \mathbf{I}_{\left\{x_{2} \geqslant D_{2}\right\}}\right] \leqslant \frac{\mathbb{E}\left[x_{1}^{\eta_{1}} x_{2}^{\eta_{2}}\right]}{D_{1}^{\eta_{1}} D_{2}^{\eta_{2}}} \leqslant \frac{e^{c_{1} / c_{2}}}{D_{1}^{\eta_{1}} D_{2}^{\eta_{2}}},
\end{aligned}
$$

where $\mathbf{I}$ is the indicator function. From the biological point of view, this implies that it is unlikely that the amount of two populations will become very large simultaneously.

\subsection{Asymptotic pathwise estimation}

Theorem 3.4. For any initial value $x_{0} \in \mathbb{R}_{+}^{2}$, the solution of model (1.3) has the property that

$$
\limsup _{t \rightarrow \infty} \frac{\log |x(t)|}{\log t} \leqslant 6 \quad \text { a.s. }
$$

Proof. Defining V: $\mathbb{R}_{+}^{2} \rightarrow \mathbb{R}_{+}$by $V(x)=x_{1}+x_{2}$, for any constant $\gamma>0$ we obtain

$$
e^{\gamma t} \log V(x(t))=\log V(x(0))+\int_{0}^{t} e^{\gamma u} g(x(u)) d u+\sum_{i=1}^{6} \breve{M}_{i}(t),
$$

where

$$
\begin{aligned}
g(x) & =\gamma \log V(x)+\frac{1}{V(x)}\left(a x_{1}-c x_{2}-b x_{1}^{2}-f x_{2}^{2}-\frac{s x_{1} x_{2}}{\beta+x_{2}}+\frac{h x_{1} x_{2}}{\beta+x_{2}}\right)-\frac{1}{2 V^{2}(x)}\left(\sigma_{1}^{2} x_{1}^{2}+\sigma_{2}^{2} x_{2}^{2}\right. \\
& +\frac{r_{1}^{2} x_{1}^{2} x_{2}^{2}}{\left(\beta+x_{2}\right)^{2}}+\frac{r_{2}^{2} x_{1}^{2} x_{2}^{2}}{\left(\beta+x_{2}\right)^{2}}+\delta_{1}^{2} x_{1}^{4}+\delta_{2}^{2} x_{2}^{4}-2 \sigma_{1} \sigma_{2} \rho_{12} x_{1} x_{2}+\frac{2 \sigma_{1} r_{2} \rho_{14} x_{1}^{2} x_{2}}{\beta+x_{2}}-2 \sigma_{1} \delta_{2} \rho_{16} x_{1} x_{2}^{2} \\
& +\frac{2 r_{1} \sigma_{2} \rho_{23} x_{1} x_{2}^{2}}{\beta+x_{2}}-\frac{2 r_{1} r_{2} \rho_{34} x_{1}^{2} x_{2}^{2}}{\left(\beta+x_{2}\right)^{2}}+\frac{2 r_{1} \delta_{2} \rho_{36} x_{1} x_{2}^{3}}{\beta+x_{2}}+2 \delta_{1} \sigma_{2} \rho_{25} x_{1}^{2} x_{2}-\frac{2 \delta_{1} r_{2} \rho_{45} x_{1}^{3} x_{2}}{\beta+x_{2}} \\
& \left.+2 \delta_{1} \delta_{2} \rho_{56} x_{1}^{2} x_{2}^{2}\right)
\end{aligned}
$$

and

$$
\begin{aligned}
& \breve{M}_{1}(t)=\sigma_{1} \int_{0}^{t} \frac{e^{\gamma u} x_{1}(u)}{V(x(u))} d B_{1}(u), \quad \breve{M}_{2}(t)=-\sigma_{2} \int_{0}^{t} \frac{e^{\gamma u} x_{2}(u)}{V(x(u))} d B_{2}(u), \\
& \breve{M}_{3}(t)=-r_{1} \int_{0}^{t} \frac{e^{\gamma u} x_{1}(u) x_{2}(u)}{\left(\beta+x_{2}(u) V(x(u))\right.} d B_{3}(u), \\
& \breve{M}_{4}(t)=r_{2} \int_{0}^{t} \frac{e^{\gamma u} x_{1}(u) x_{2}(u)}{\left(\beta+x_{2}(u)\right) V(x(u))} d B_{4}(u), \\
& \breve{M}_{5}(t)=-\delta_{1} \int_{0}^{t} \frac{e^{\gamma u} x_{1}^{2}(u)}{V(x(u))} d B_{5}(u), \quad \breve{M}_{6}(t)=-\delta_{2} \int_{0}^{t} \frac{e^{\gamma u} x_{2}^{2}(u)}{V(x(u))} d B_{6}(u)
\end{aligned}
$$


are local martingales with quadratic variations

$$
\begin{aligned}
& \left\langle\breve{M}_{1}(t)\right\rangle=\sigma_{1}^{2} \int_{0}^{t} \frac{e^{2 \gamma u} x_{1}^{2}(u)}{V^{2}(x(u))} d u, \quad\left\langle\breve{M}_{2}(t)\right\rangle=\sigma_{2}^{2} \int_{0}^{t} \frac{e^{2 \gamma u} x_{1}^{2}(u)}{V^{2}(x(u))} d u, \\
& \left\langle\breve{M}_{3}(t)\right\rangle=r_{1}^{2} \int_{0}^{t} \frac{e^{2 \gamma u} x_{1}^{2}(u) x_{2}^{2}(u)}{\left(\beta+x_{2}(u)\right)^{2} V^{2}(x(u))} d u, \quad\left\langle\breve{M}_{4}(t)\right\rangle=r_{2}^{2} \int_{0}^{t} \frac{e^{2 \gamma u} x_{1}^{2}(u) x_{2}^{2}(u)}{\left(\beta+x_{2}(u)\right)^{2} V^{2}(x(u))} d u, \\
& \left\langle\breve{M}_{5}(t)\right\rangle=\delta_{1}^{2} \int_{0}^{t} \frac{e^{2 \gamma u} x_{1}^{4}(u)}{V^{2}(x(u))} d u, \quad\left\langle\breve{M}_{6}(t)\right\rangle=\delta_{2}^{2} \int_{0}^{t} \frac{e^{2 \gamma u} x_{2}^{4}(u)}{V^{2}(x(u))} d u .
\end{aligned}
$$

Given any $\alpha_{1} \in(0,1)$ and $p>1$. By the exponential martingale inequality, we have

$$
\mathbb{P}\left(\sup _{0 \leqslant t \leqslant \psi}\left(\breve{M}_{i}(t)-\frac{\alpha_{1}}{2} e^{-\gamma \psi}\left\langle\breve{M}_{i}(t)\right\rangle\right)>\frac{p e^{\gamma \psi}}{\alpha_{1}} \log \psi\right) \leqslant \frac{1}{\psi^{p}}, \quad \psi=1,2, \cdots
$$

Then by the Borel-Cantelli lemma, for almost all $\omega \in \Omega$, there exists $\psi_{i}=\psi_{i}(\omega) \geqslant 1$ such that

$$
\breve{M}_{i}(t) \leqslant \frac{\alpha_{1}}{2} e^{-\gamma \psi}\left\langle\breve{M}_{i}(t)\right\rangle+\frac{p e^{\gamma \psi}}{\alpha_{1}} \log \psi \text { for all } 0 \leqslant t \leqslant \psi \text { and } \psi \geqslant \psi_{i} .
$$

Thus substituting this into (3.8) indicates that for almost every $\omega \in \Omega$,

$$
\begin{aligned}
& e^{\gamma t} \log V(x(t)) \\
\leqslant & \log V(x(0))+\int_{0}^{t} e^{\gamma u}\left(\gamma \log V(x(u))+a+h+g_{1}(x(u))-\frac{1-\alpha_{1} e^{\gamma(u-\psi)}}{2 V^{2}(x(u))}\left(\delta_{1}^{2} x_{1}^{4}(u)+\delta_{2}^{2} x_{2}^{4}(u)\right)\right) d u \\
& +\frac{6 p e^{\gamma \psi}}{\alpha_{1}} \log \psi
\end{aligned}
$$

for all $0 \leqslant t \leqslant \psi$ and $\psi \geqslant \psi_{0}:=\max \left(\psi_{1}, \psi_{2}, \cdots, \psi_{6}\right)$, where $g_{1}(x)$ is a first-order polynomial about $x$. By the elementary inequality

$$
\frac{V^{2}(x)}{2} \leqslant|x|^{2} \leqslant 2 V^{2}(x)
$$

we obtain

$$
\frac{1}{V^{2}(x)}\left(\delta_{1}^{2} x_{1}^{4}+\delta_{2}^{2} x_{2}^{4}\right) \geqslant \frac{1}{4}\left(\delta_{1}^{2} \wedge \delta_{2}^{2}\right)|x|^{2} .
$$

Therefore (3.9) is rewritten as

$$
\begin{aligned}
& e^{\gamma t} \log V(x(t)) \\
\leqslant & \log V(x(0))+\int_{0}^{t} e^{\gamma u}\left(\gamma \log V(x(u))+a+h+g_{1}(x(u))-\frac{1}{8}\left(1-\alpha_{1}\right)\left(\delta_{1}^{2} \wedge \delta_{2}^{2}\right)|x(u)|^{2}\right) d u \\
& +\frac{6 p e^{\gamma \psi}}{\alpha_{1}} \log \psi .
\end{aligned}
$$

Obviously, there exists a positive constant $K_{3}$ such that for almost every $\omega \in \Omega$,

$$
\begin{aligned}
& e^{\gamma t} \log V(x(t)) \\
\leqslant & \log V(x(0))+K_{3} \int_{0}^{t} e^{\gamma u} d u+\frac{6 p e^{\gamma \psi}}{\alpha_{1}} \log \psi \leqslant \log V(x(0))+\frac{K_{3}}{\gamma} e^{\gamma t}-\frac{K_{3}}{\gamma}+\frac{6 p e^{\gamma \psi}}{\alpha_{1}} \log \psi
\end{aligned}
$$

for all $0 \leqslant t \leqslant \psi$ and $\psi \geqslant \psi_{0}:=\max \left(\psi_{1}, \psi_{2}, \cdots, \psi_{6}\right)$. Consequently, for $\psi-1 \leqslant t \leqslant \psi$ and $\psi \geqslant \psi_{0}$, it follows that

$$
\frac{\log V(x(t))}{\log t} \leqslant \frac{1}{\log (\psi-1)}\left(e^{-\gamma(\psi-1)} \log \left(x_{1}(0) x_{2}(0)\right)+\frac{K_{3}}{\gamma}+\frac{6 p e^{\gamma}}{\alpha_{1}} \log \psi\right) .
$$


This implies

$$
\limsup _{t \rightarrow \infty} \frac{\log V(x(t))}{\log t} \leqslant \frac{6 p e^{\gamma}}{\alpha_{1}} \quad \text { a.s. }
$$

Letting $\alpha_{1} \rightarrow 1, p \rightarrow 1$ and $\gamma \rightarrow 0$ implies

$$
\limsup _{t \rightarrow \infty} \frac{\log V(x(t))}{\log t} \leqslant 6 \quad \text { a.s. }
$$

Recalling inequality (3.10) gives the required assertion (3.7).

Remark 3.5. Let all the Brownian motions in model (1.3) be uncorrelated. Then Theorem 3.4 still holds. Besides, Theorem 3.2 is fulfilled provided that $\eta_{1}$ and $\eta_{2}$ satisfy

$$
\eta_{1}, \eta_{2}<\frac{1}{2}
$$

with $c_{1}$ and $c_{2}$ defined by

$$
c_{1}=\frac{\left(h \eta_{2} / \beta-b \eta_{1}\right)^{2}+\eta_{1} \delta_{1}^{2}\left(1-\eta_{1}\right)\left(a \eta_{1}-c \eta_{2}\right)}{\eta_{1}\left(1-\eta_{1}\right) \delta_{1}^{2}}
$$

and

$$
c_{2}=\frac{1}{4}\left(\eta_{1}\left(1-\eta_{1}\right) \delta_{1}^{2} \wedge \eta_{2}\left(1-\eta_{2}\right) \delta_{2}^{2}\right)
$$

\section{Stationary distribution}

In this section, the stationary distributions of the solutions of model (1.2) and (1.3) are established. Let $P_{x_{0}, t}$ denote the probability measure induced by $x(t)$ with initial value $x(0)=x_{0}$, that is

$$
P_{x_{0}, t}(D)=\mathbb{P}(x(t) \in D), \quad D \in \mathcal{B}\left(\mathbb{R}_{+}^{2}\right),
$$

where $\mathcal{B}\left(\mathbb{R}_{+}^{2}\right)$ is the $\sigma$-algebra of all the Borel sets $D \subseteq \mathbb{R}_{+}^{2}$. If there is a probability measure $\mu(\cdot)$ on the measurable space $\left(\mathbb{R}_{+}^{2}, \mathcal{B}\left(\mathbb{R}_{+}^{2}\right)\right)$ such that

$$
P_{x_{0}, t}(\cdot) \rightarrow \mu(\cdot) \text { in distribution for any } x_{0} \in \mathbb{R}_{+}^{2},
$$

we then say that the SDE model (1.2) (or (1.3)) has a stationary distribution $\mu(\cdot)[23,31,41]$. To show the existence of a stationary distribution, let us first cite a known result from Khasminskii [41, pp. 107-109, Theorem 4.1] as a lemma.

Lemma 4.1. The SDE model (1.3) has a unique stationary distribution if

(i) the matrix

$$
U(x)=A(x) \mathcal{R} A(x)^{T}
$$

is positive definite for $x \in \mathbb{R}_{+}^{2}$, where

$$
A(x)=\left[\begin{array}{cccccc}
\sigma_{1} x_{1} & 0 & \frac{-r_{1} x_{1} x_{2}}{\beta+x_{2}} & 0 & -\delta_{1} x_{1}^{2} & 0 \\
0 & -\sigma_{2} x_{2} & 0 & \frac{r_{2} x_{1} x_{2}}{\beta+x_{2}} & 0 & -\delta_{2} x_{2}^{2}
\end{array}\right]
$$

(ii) there is a bounded openset $G$ of $\mathbb{R}_{+}^{2}$ and

$$
\sup _{x_{0} \in Q-G} \mathbb{E}\left(\tau_{G}\right)<\infty
$$

for every compact subset $Q$ of $\mathbb{R}_{+}^{2}$ such that $G \subset Q$ where $\tau_{G}=\inf \{t \geqslant 0: x(t) \in G\}$. 
Theorem 4.2. If

$$
\begin{gathered}
\rho_{i_{1} i_{2}} \neq \pm 1 ; \rho_{1 i_{3}}, \rho_{4 i_{3}}<1 / 2 ; \rho_{26}, \rho_{35}>-1 / 2 ; \\
\rho_{1 i_{3}} \leqslant \rho_{1 i_{2}} \rho_{i_{2} i_{3}} ; \rho_{35} \geqslant \rho_{3 i_{2}} \rho_{5 i_{2}} ; \rho_{4 i_{4}} \leqslant \rho_{i_{1} 4} \rho_{i_{1} i_{4}} ; \rho_{26} \geqslant \rho_{2 i_{1}} \rho_{6 i_{1}} ; \\
2 \rho_{1 i_{3}} \rho_{26} \leqslant \rho_{12} \rho_{6 i_{3}}+\rho_{16} \rho_{2 i_{3}} ; 2 \rho_{35} \rho_{4 i_{4}} \leqslant \rho_{3 i_{4}} \rho_{45}+\rho_{34} \rho_{5 i_{4}} ; \\
2 \rho_{1 i_{3}} \rho_{4 i_{4}} \geqslant \rho_{14} \rho_{i_{3} i_{4}}+\rho_{1 i_{4}} \rho_{4 i_{3}} ; 2 \rho_{35} \rho_{26} \geqslant \rho_{23} \rho_{56}+\rho_{36} \rho_{25} \\
\text { for } i_{1}=1,3 \text { or } 5, i_{2}=2,4 \text { or } 6, i_{3}=3 \text { or } 5 \text { and } i_{4}=2 \text { or } 6, \\
h_{0}:=\sigma_{2} r_{2} \tilde{\rho}_{24}+\delta_{2} r_{2} \beta \tilde{\rho}_{46}<h, \\
2 a\left(1-\frac{1+\beta}{2\left(h-h_{0}\right)} \sigma_{2}^{2}-\frac{(1+\beta) c}{h-h_{0}}\right)>\sigma_{1}^{2}+2 r_{1} \sigma_{1} \tilde{\rho}_{13}+\frac{2\left(b+b_{0}\right) \beta c}{h-h_{0}}+\frac{\left(b+b_{0}\right) \beta}{h-h_{0}} \sigma_{2}^{2}
\end{gathered}
$$

and

$$
b \geqslant \frac{1}{2} \delta_{1}^{2}+\frac{E_{1}}{2 h \beta^{2}} r_{2}^{2}+\frac{a+E_{2}}{\beta}
$$

where

$$
b_{0}=\sigma_{1} \delta_{1} \tilde{\rho}_{15}+r_{1} \delta_{1} \bar{\rho}_{35}, \quad E_{1}=\frac{a h+\left(a+b+b_{0}\right) \beta h}{h-h_{0}} \quad \text { and } \quad E_{2}=b_{0}+\frac{h_{0} E_{1}}{\beta h},
$$

then for any initial value $x_{0} \in \mathbb{R}_{+}^{2}$, model (1.3) has a unique stationary distribution.

Proof. (i) We compute

$$
U(x)=\left(U_{i j}(x)\right)_{2 \times 2},
$$

where

$$
\begin{aligned}
& U_{11}(x)=\sigma_{1}^{2} x_{1}^{2}-\frac{2 \rho_{13} \sigma_{1} r_{1} x_{1}^{2} x_{2}}{\beta+x_{2}}-2 \sigma_{1} \delta_{1} \rho_{15} x_{1}^{3}+\frac{2 r_{1} \delta_{1} \rho_{35} x_{1}^{3} x_{2}}{\beta+x_{2}}+\frac{r_{1}^{2} x_{1}^{2} x_{2}^{2}}{\left(\beta+x_{2}\right)^{2}}+\delta_{1}^{2} x_{1}^{4}, \\
& U_{22}(x)=\sigma_{2}^{2} x_{2}^{2}-\frac{2 r_{2} \sigma_{2} \rho_{24} x_{1} x_{2}^{2}}{\beta+x_{2}}+2 \rho_{26} \sigma_{2} \delta_{2} x_{2}^{3}-\frac{2 \rho_{46} \delta_{2} r_{2} x_{1} x_{2}^{3}}{\beta+x_{2}}+\frac{r_{2}^{2} x_{1}^{2} x_{2}^{2}}{\left(\beta+x_{2}\right)^{2}}+\delta_{2}^{2} x_{2}^{4}
\end{aligned}
$$

and

$$
\begin{aligned}
U_{12}(x) & =U_{21}(x)=-\sigma_{1} \sigma_{2} \rho_{21} x_{1} x_{2}+\frac{\rho_{14} r_{2} \sigma_{1} x_{1}^{2} x_{2}}{\beta+x_{2}}-\delta_{2} \sigma_{1} \rho_{16} x_{1} x_{2}^{2}+\frac{r_{1} \sigma_{2} \rho_{23} x_{1} x_{2}^{2}}{\beta+x_{2}} \\
& -\frac{\rho_{34} r_{1} r_{2} x_{1}^{2} x_{2}^{2}}{\left(\beta+x_{2}\right)^{2}}+\frac{r_{2} \delta_{2} \rho_{36} x_{1} x_{2}^{3}}{\beta+x_{2}}+\delta_{1} \sigma_{2} \rho_{25} x_{1}^{2} x_{2}-\frac{\rho_{45} \delta_{1} r_{2} x_{1}^{3} x_{2}}{\beta+x_{2}}+\rho_{56} \delta_{1} \delta_{2} x_{1}^{2} x_{2}^{2} .
\end{aligned}
$$

The sufficient conditions (4.1) guarantee that $U_{11}(x)>0, U_{22}(x)>0$ and $U_{11}(x) U_{22}(x)-U_{12}^{2}(x)>0$. Hence $U(x)$ is a positive-definite matrix.

(ii) We define a $C^{2}$-function $V: \mathbb{R}_{+}^{2} \rightarrow \mathbb{R}_{+}$:

$$
V(x)=M V_{1}(x)+V_{2}(x)+e,
$$

where

$$
V_{1}(x)=x_{1}+\ln \left(\beta+x_{1}\right)-\ln \left(x_{1}\right)+\frac{l}{h} x_{2}-\frac{E_{1}}{h} \ln x_{2}, \quad V_{2}(x)=x_{1}+\frac{s}{h} x_{2},
$$

and $e, l$ and $M$ are three constants. $e=-\min \left(M V_{1}(x)+V_{2}(x)\right)$ to keep the non-negativity of $V(x)$,

$$
l=\left(\frac{h s}{c \beta}+\frac{E_{1} f}{c}+\frac{E_{1} \sigma_{2} \delta_{2} \bar{\rho}_{26}}{c}\right) \bigvee\left(\frac{E_{1} \delta_{2}^{2}}{2 f}+\frac{h r_{1}^{2}}{2 f \beta^{2}}+\frac{E_{1} h}{4 f \beta^{2}}\right)
$$


and $M$ is to be defined later. First compute

$$
\begin{aligned}
L V_{1} & =\left(x_{1}+\frac{x_{1}}{\beta+x_{1}}-1\right)\left(a-b x_{1}-\frac{s x_{2}}{\beta+x_{2}}\right)+\frac{1}{2}\left(1-\frac{x_{1}^{2}}{\left(\beta+x_{1}\right)^{2}}\right)\left(\sigma_{1}^{2}+\frac{r_{1}^{2} x_{2}^{2}}{\left(\beta+x_{2}\right)^{2}}+\delta_{1}^{2} x_{1}^{2}\right. \\
& \left.-\frac{2 \sigma_{1} r_{1} \rho_{13} x_{2}}{\beta+x_{2}}-2 \sigma_{1} \delta_{1} \rho_{15} x_{1}+\frac{2 r_{1} \delta_{1} \rho_{35} x_{1} x_{2}}{\beta+x_{2}}\right)+\left(\frac{l x_{2}}{h}-\frac{E_{1}}{h}\right)\left(\frac{h x_{1}}{\beta+x_{2}}-c-f x_{2}\right) \\
& +\frac{E_{1}}{2 h}\left(\sigma_{2}^{2}+\frac{r_{2}^{2} x_{1}^{2}}{\left(\beta+x_{2}\right)^{2}}+\delta_{2}^{2} x_{2}^{2}-\frac{2 r_{2} \sigma_{2} \rho_{24} x_{1}}{\beta+x_{2}}+2 \delta_{2} \sigma_{2} \rho_{26} x_{2}-\frac{2 \delta_{2} r_{2} \rho_{46} x_{1} x_{2}}{\beta+x_{2}}\right) \\
& \leqslant a x_{1}-b x_{1}^{2}+\frac{a x_{1}}{\beta+x_{1}}-\frac{b x_{1}^{2}}{\beta+x_{1}}+b x_{1}-\frac{E_{1} x_{1}}{\beta+x_{2}}-a+\frac{s x_{2}}{\beta+x_{2}}+\frac{1}{2} \sigma_{1}^{2}+\frac{r_{1}^{2} x_{2}^{2}}{2 \beta^{2}}+\frac{\delta_{1}^{2} x_{1}^{2}}{2} \\
& +r_{1} \sigma_{1} \tilde{\rho}_{13}+\left(\sigma_{1} \delta_{1} \tilde{\rho}_{15}+r_{1} \delta_{1} \bar{\rho}_{35}\right) x_{1}+\frac{l x_{1} x_{2}}{\beta+x_{2}}-\frac{c l x_{2}}{h}-\frac{f l x_{2}^{2}}{h}+\frac{E_{1} c}{h}+\frac{E_{1} f x_{2}}{h}+\frac{E_{1} \sigma_{2}^{2}}{2 h} \\
& +\frac{E_{1} r_{2}^{2} x_{1}^{2}}{2 h \beta^{2}}+\frac{E_{1} \delta_{2}^{2} x_{2}^{2}}{2 h}+\frac{E_{1} \sigma_{2} r_{2} \tilde{\rho}_{24} x_{1}}{h \beta}+\frac{E_{1} \sigma_{2} \delta_{2} \rho_{26} x_{2}}{h}+\frac{E_{1} \delta_{2} r_{2} \tilde{\rho}_{46} x_{1}}{h} \\
& \leqslant \frac{\left(a+E_{2}\right) \beta x_{1}}{\beta+x_{1}}+\frac{a x_{1}}{\beta+x_{1}}+\frac{b \beta x_{1}}{\beta+x_{1}}-\frac{E_{1} x_{1}}{\beta+x_{2}}+\left(-b+\frac{\delta_{1}^{2}}{2}+\frac{a+E_{2}}{\beta+x_{1}}+\frac{E_{1} r_{2}^{2}}{2 h \beta^{2}}\right) x_{1}^{2} \\
& -a+\frac{1}{2} \sigma_{1}^{2}+r_{1} \sigma_{1} \tilde{\rho}_{13}+\frac{E_{1} c}{h}+\frac{E_{1} \sigma_{2}^{2}}{2 h}+\left(\frac{s}{\beta}+\frac{E_{1} f}{h}-\frac{c l}{h}+\frac{E_{1} \sigma_{2} \delta_{2} \rho_{26}}{h}\right) x_{2}+\left(\frac{E_{1} \delta_{2}^{2}}{2 h}+\frac{r_{1}^{2}}{2 \beta^{2}}\right. \\
& \left.-\frac{f l}{h}\right) x_{2}^{2}+\frac{l x_{1} x_{2}}{\beta+x_{2}} \\
& \leqslant \frac{E_{1}\left(x_{1} x_{2}-x_{1}^{2}\right)}{\left(\beta+x_{1}\right)\left(\beta+x_{2}\right)}+\left(-b+\frac{\delta_{1}^{2}}{2}+\frac{a+E_{2}}{\beta}+\frac{E_{1} r_{2}^{2}}{2 h \beta^{2}}\right) x_{1}^{2}-\lambda+\left(\frac{s}{\beta}+\frac{E_{1} f}{h}-\frac{c l}{h}\right. \\
& \left.+\frac{E_{1} \sigma_{2} \delta_{2} \rho_{26}}{h}\right) x_{2}+\left(\frac{E_{1} \delta_{2}^{2}}{2 h}+\frac{r_{1}^{2}}{2 \beta^{2}}-\frac{f l}{h}\right) x_{2}^{2}+\frac{l x_{1} x_{2}}{\beta+x_{2}},
\end{aligned}
$$

where $\lambda=a-\frac{1}{2} \sigma_{1}^{2}-r_{1} \sigma_{1} \tilde{\rho}_{13}-\frac{E_{1} c}{h}-\frac{E_{1} \sigma_{2}^{2}}{2 h}>0$ from condition (4.3). Under (4.4), (4.5) and the Young inequality,

$$
\begin{aligned}
L V_{1} & \leqslant-\lambda+\left(-b+\frac{\delta_{1}^{2}}{2}+\frac{a+E_{2}}{\beta}+\frac{E_{1} r_{2}^{2}}{2 h \beta^{2}}\right) x_{1}^{2}+\left(\frac{s}{\beta}+\frac{E_{1} f}{h}-\frac{c l}{h}+\frac{E_{1} \sigma_{2} \delta_{2} \rho_{26}}{h}\right) x_{2} \\
& +\left(\frac{E_{1} \delta_{2}^{2}}{2 h}+\frac{r_{1}^{2}}{2 \beta^{2}}-\frac{f l}{h}+\frac{E_{1}}{4 \beta^{2}}\right) x_{2}^{2}+\frac{l x_{1} x_{2}}{\beta+x_{2}} \\
& \leqslant-\lambda+\frac{l x_{1} x_{2}}{\beta+x_{2}} .
\end{aligned}
$$

Then compute

$$
L V_{2}=a x_{1}-b x_{1}^{2}-\frac{s c}{h} x_{2}-\frac{s f}{h} x_{2}^{2} \leqslant a x_{1}-b x_{1}^{2}-\frac{s f}{h} x_{2}^{2}
$$

Hence

$$
L V(x) \leqslant M\left(-\lambda+\frac{l x_{1} x_{2}}{\beta+x_{2}}\right)+a x_{1}-b x_{1}^{2}-\frac{s f}{h} x_{2}^{2},
$$

where $M$ satisfies $M \lambda \geqslant a^{2} /(4 b)+2$. Now we aim to show

$$
L V(x) \leqslant-1 \text { for all } x \in \mathbb{R}_{+}^{2}-G:=G^{c} .
$$

As if this holds, let $x \in G^{c}$ be arbitrary and $\tau_{G}$ be the stopping time as defined in Lemma 4.1. From (4.6), we have

$$
0 \leqslant V\left(x_{0}\right)-\mathbb{E}\left(t \wedge \tau_{G} \wedge \tau_{k}\right), \quad \forall t>0 .
$$

Letting $k \rightarrow \infty$ and then $t \rightarrow \infty$, we have

$$
\mathbb{E}\left(\tau_{G}\right) \leqslant V\left(x_{0}\right), \quad \forall x_{0} \in G^{c}
$$


as required. To show that (4.6) actually holds, we define

$$
G^{c}=G_{1}^{c} \cup G_{2}^{c} \cup G_{3}^{c} \cup G_{4}^{c},
$$

where

$$
\begin{aligned}
& G_{1}^{c}=\left\{x \mid x_{1} \in\left(0, \epsilon_{1}\right]\right\} ; \quad G_{2}^{c}=\left\{x \mid x_{1} \in\left(0, \frac{1}{\epsilon_{1}}\right], x_{2} \in\left(0, \epsilon_{2}\right]\right\} \\
& G_{3}^{c}=\left\{x \mid x_{1} \in\left[\frac{1}{\epsilon_{1}},+\infty\right)\right\} ; \quad G_{4}^{c}=\left\{x \mid x_{2} \in\left[\frac{1}{\epsilon_{2}},+\infty\right)\right\}
\end{aligned}
$$

with two constants $\epsilon_{1}, \epsilon_{2} \in(0,1)$ satisfying

$$
\epsilon_{1}^{2} \leqslant \frac{1}{M^{2} l^{2}} \bigwedge \frac{b}{2\left(N_{1}+1\right)}, \quad \epsilon_{2}^{2} \leqslant \frac{s f}{2 h\left(N_{2}+1\right)} \quad \text { and } \quad \epsilon_{2} \leqslant \frac{\beta \epsilon_{1}}{M l},
$$

where the constants $N_{1}$ and $N_{2}$ will be determined later. We then show that in any subset of $G^{c},(4.6)$ holds. From (4.7),

(a) if $x \in G_{1}^{c}$,

$$
L V(x) \leqslant-M \lambda+M l x_{1}+a x_{1}-b x_{1}^{2}-\frac{s f}{h} x_{2}^{2} \leqslant M l \epsilon_{1}-2 \leqslant-1 ;
$$

(b) if $x \in G_{2}^{c}$,

$$
L V(x) \leqslant-M \lambda+\frac{M l x_{1} x_{2}}{\beta}+a x_{1}-b x_{1}^{2}-\frac{s f}{h} x_{2}^{2} \leqslant \frac{M l \epsilon_{2}}{\beta \epsilon_{1}}-2 \leqslant-1
$$

(c) if $x \in G_{3}^{c}$,

$$
L V(x) \leqslant-M \lambda+(M l+a) x_{1}-\frac{b x_{1}^{2}}{2}-\frac{b x_{1}^{2}}{2}-\frac{s f x_{2}^{2}}{h} .
$$

Note that the polynomials $-M \lambda+(M l+a) x_{1}-\frac{b x_{1}^{2}}{2}-\frac{s f x_{2}^{2}}{h}$ has an upper bound, say $N_{1}$, hence

$$
L V(x) \leqslant N_{1}-\frac{b}{2 \epsilon_{1}^{2}} \leqslant-1
$$

(d) if $x \in G_{4}^{c}$,

$$
L V(x) \leqslant-M \lambda+(M l+a) x_{1}-b x_{1}^{2}-\frac{s f x_{2}^{2}}{2 h}-\frac{s f x_{2}^{2}}{2 h} .
$$

Note that the polynomial $-M \lambda+(M l+a) x_{1}-b x_{1}^{2}-\frac{s f x_{2}^{2}}{2 h}$ is again bounded, say by $N_{2}$, we have

$$
L V(x) \leqslant N_{2}-\frac{s f}{2 h \epsilon_{2}^{2}} \leqslant-1 .
$$

In all,

$$
L V(x) \leqslant-1 \text { for all } x \in G^{c} .
$$

Remark 4.3. Assume that all the Brownian motions in model (1.3) are uncorrelated. Then letting $\rho_{i j}=0$ for $i, j=1,2, \cdots, 6$ and $i \neq j$ in condition (4.2)-(4.4) gives the parametric conditions of model (1.3) to have a unique stationary distribution.

Remark 4.4. Given that condition (4.2)-(4.4) are satisfied with $\delta_{1}=\delta_{2}=0$ and $\rho_{i 5}=\rho_{i 6}=0$ for $i=1,2, \cdots, 4$, model (1.2) then has a unique stationary distribution. 


\section{Simulations}

The following examples are developed to illustrate our results. The system parameters are given in appropriate units. The Euler-Maruyama (EM) scheme is used for the computer simulations [42]. From the theory introduced in [43], the EM approximate solutions are convergent to the true solutions of model (1.2) and (1.3) in probability.

Example 5.1. We perform a computer simulation of 10000 iterations of model (1.2) with initial value $x(0)=(0.7,0.15)^{T}$ using the Euler-Maruyama (EM) method [40, 42] with stepsize $\Delta=0.01$ and the system parameters given by

$$
\begin{aligned}
& a=1, b=0.5, \beta=5, s=16, h=0.9, c=2, f=3, \sigma_{1}=1.5, \sigma_{2}=1.0, r_{1}=0.5 \\
& r_{2}=0.95 \text { and } \rho_{13}=0.15
\end{aligned}
$$

This group of parameters satisfies condition (2.5) clearly. Theorem 2.4 then indicates that both species die out ultimately with probability 1 . This is illustrated in Figure 1.

Example 5.2. We keep the system parameters of model (1.2) the same as Example 5.1 but let $\sigma_{1}=0.5$. Moreover, we let $\rho_{13}=0.15$ and $\rho_{24}=0.9$. As a result, condition (2.6) is fulfilled. From Theorem 2.4(b), the prey abundance has the property that

$$
1.75 \leqslant \liminf _{t \rightarrow \infty} \frac{1}{t} \int_{0}^{t} x_{1}(u) d u \leqslant \limsup _{t \rightarrow \infty} \frac{1}{t} \int_{0}^{t} x_{1}(u) d u \leqslant 1.825 \quad \text { a.s. }
$$

and the consumers will tend to zero exponentially with probability 1 . Figure 2 supports these results clearly.

Example 5.3. In this example, we remain the system parameters of model (1.2) the same as Example 5.2 except that we let $\rho_{24}=-0.95$. This group of parameters does not obey condition (2.6) but satisfy (2.7). Hence Theorem 2.4(b) suggests that

$$
1.75 \leqslant \liminf _{t \rightarrow \infty} \frac{1}{t} \int_{0}^{t} x_{1}(u) d u \leqslant \limsup _{t \rightarrow \infty} \frac{1}{t} \int_{0}^{t} x_{1}(u) d u \leqslant 1.825 \quad \text { a.s. }
$$

and the consumers will tend to zero exponentially with probability 1 . Figure 3 supports these results clearly.

Then we study the case when the SDE system (1.2) and (1.3) have a stationary distribution.

Example 5.4. We assume that the parameters of system (1.2) are the same as in Example 5.3 but let $\beta=2.5, h=10, \sigma_{1}=0.01$, and $\sigma_{2}=0.02$. Also the correlation matrix is given by

$$
\mathcal{R}=\left(\rho_{i j}\right)_{4 \times 4}=\left[\begin{array}{cccc}
1 & 0 & -0.8 & 0 \\
0 & 1 & 0 & -0.95 \\
-0.8 & 0 & 1 & 0 \\
0 & -0.95 & 0 & 1
\end{array}\right]
$$

The time series of the correlated Brownian motions is shown in Figure 4. It is found that these parameters obey conditions (4.1)-(4.4) with $\delta_{1}=\delta_{2}=0$ and $\rho_{i 5}=\rho_{i 6}=0=\rho_{56}$ for $i=1,2, \cdots, 4$. From Theorem 2.4 and Remark 4.4, system (1.2) has a stationary distribution. The ergodic property enables us to obtain the approximate probability distribution for the stationary distribution by computer simulation of a single sample path of a solution to model (1.2). Therefore the histogram of the 10000 iterations shown in Figure 5(b)(d) can be regarded as approximate p.d.f.s of the stationary distribution. 
Example 5.5. In this example, the stationary distribution of model (1.3) is examined. We keep the system parameters the same as in Example 5.4 and let $\delta_{1}=0.01$ and $\delta_{2}=0.02$. Moreover the correlation matrix is given by

$$
\mathcal{R}=\left(\rho_{i j}\right)_{6 \times 6}=\left[\begin{array}{cccccc}
1 & 0 & -0.8 & 0 & -0.5 & 0 \\
0 & 1 & 0 & -0.95 & 0 & 0.7 \\
-0.8 & 0 & 1 & 0 & 0.9 & 0 \\
0 & -0.95 & 0 & 1 & 0 & -0.8 \\
-0.5 & 0 & 0.9 & 0 & 1 & 0 \\
0 & 0.7 & 0 & -0.8 & 0 & 1
\end{array}\right] .
$$

Obviously, these parameters obey conditions (4.1)-(4.4). From Theorem 4.2, model (1.3) has a stationary distribution. The approximate p.d.f.s of the stationary distribution could be identified from Figure 6(b)(d).

\section{Summary}

In this chapter, the different properties of the SDE population models (1.2) and (1.3) incorporating white noise were studied. The correlations between the Brownian motions do make an effect on the long-time behaviours of the systems. Especially, in model (1.2), a positive correlation between $B_{1}(t)$ and $B_{3}(t)$ leads to a slightly different condition for both populations to be extinct. Moreover, if $B_{2}(t)$ is strongly negatively correlated to $B_{4}(t)$, the population system always remains extinct (the prey populations become persistent while the consumers die out) and has no chance to have a multiple coexisting stationary status. In the contrast, provided that the correlation coefficient between $B_{2}(t)$ and $B_{4}(t)$ is bigger than $-\frac{h}{r_{2} \sigma_{2}}$, the system is possible to have a stationary distribution for both species with a larger value of $a$. In model (1.3), the correlations between $B_{4}(t)$ and $B_{5}(t)$ and between $B_{5}(t)$ and $B_{6}(t)$ also make a difference on the parametric conditions of the bounded properties. These imply how the correlations between the Brownian motions affect the dynamical behaviours of the populations. Theorem 4.2 reflects that a smaller amplitude environmental noise leads to a permanent population system. The ergodic property of the stationary distribution makes it possible to generate the approximate probability distribution using a single sample path of the solution to the SDE model by computer simulations.

\section{Acknowledgements \\ The authors would like to thanks the referees for their very helpful comments and suggestions.}

The first two authors would like to thank the University of Strathclyde for awarding them the PhD studentships. The first author would also like to thank China Scholarship Council and the MASTS pooling initiative (The Marine Alliance for Science and Technology for Scotland) for their financial support. MASTS is funded by the Scottish Funding Council (grant reference HR09011).

The third author would like to thank the Royal Society (WM160014, Royal Society References Wolfson Research Merit Award) and the Royal Society and the Newton Fund (NA160317, Royal Society-Newton Advanced

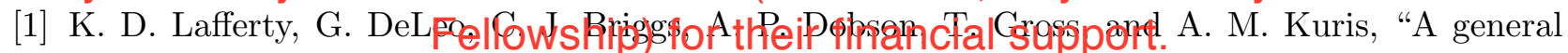
consumer-resource population model," Science, vol. 349, no. 6250, pp. 854-857, 2015.

[2] H. R. Akcakaya, R. Arditi, and L. R. Ginzburg, "Ratio-dependent predation: an abstraction that works," Ecology, vol. 76, no. 3, pp. 995-1004, 1995.

[3] M. R. Heath, D. C. Speirs, and J. H. Steele, "Understanding patterns and processes in models of trophic cascades," Ecology Letters, vol. 17, no. 1, pp. 101-114, 2014. [Online]. Available: http://doi.wiley.com/10.1111/ele.12200 


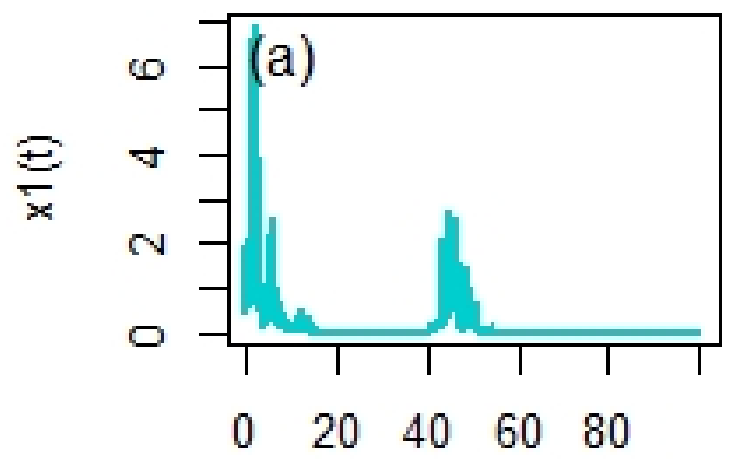

Time

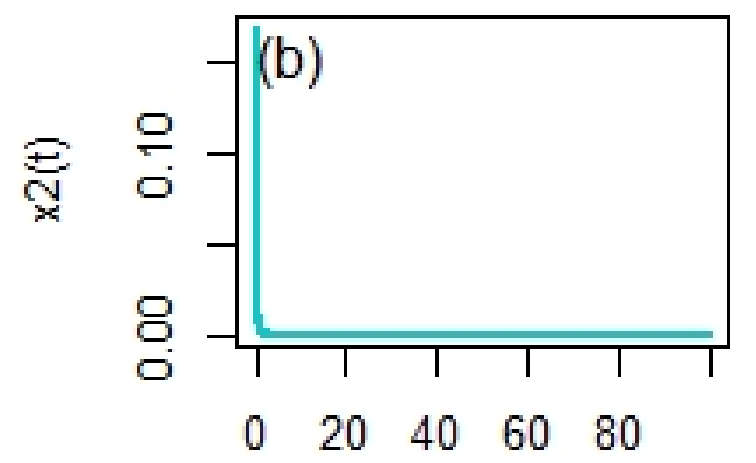

Time

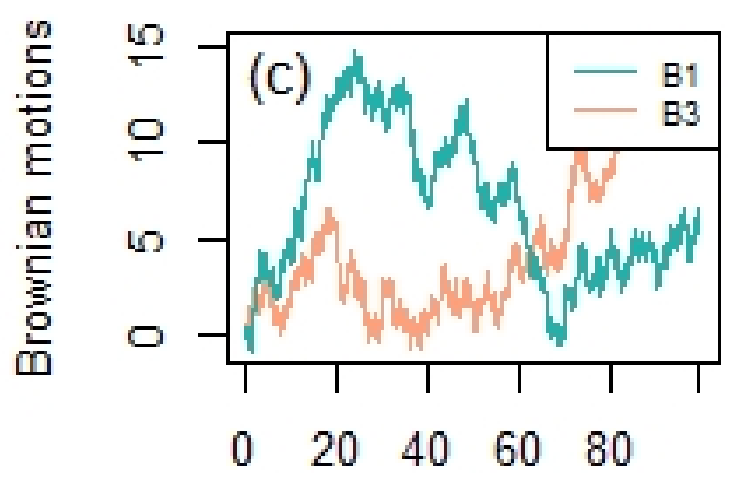

Time

Figure 1: Numerical simulations of the paths (a) $x_{1}(t)$ and (b) $x_{2}(t)$ of SDE model (1.2) using the EM scheme with stepsize $\Delta=0.01$ and initial value $x_{0}=(0.7,0.15)^{T}$ with the system parameters provided by (5.1). Times series of the correlated Brownian motions $B_{1}(t)$ and $B_{3}(t)$ is shown in (c). 


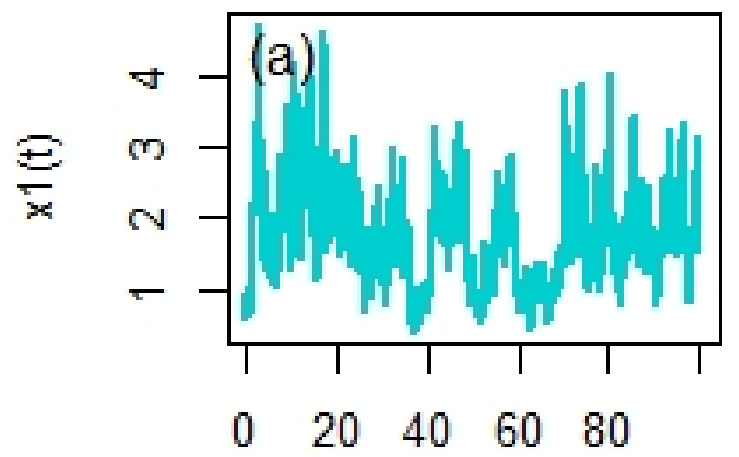

Time

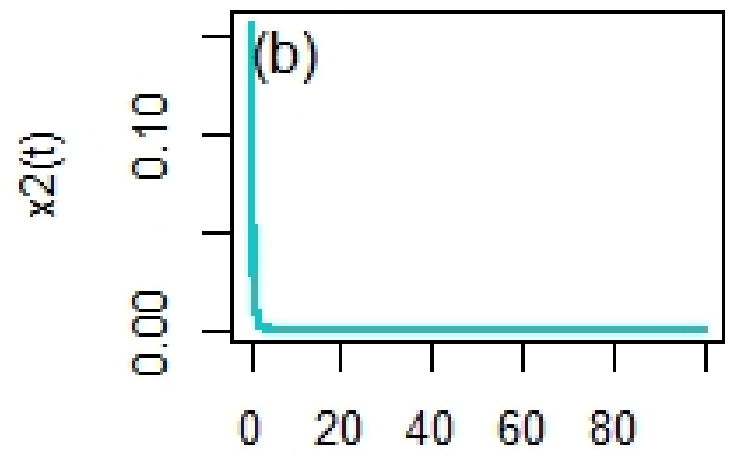

Time

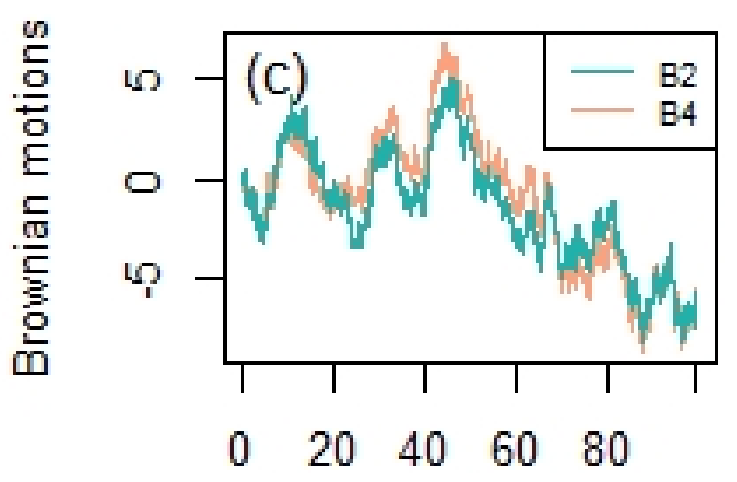

Time

Figure 2: Under the system parameters described in Example 5.2, we obtain the numerical simulations of the paths (a) $x_{1}(t)$ and (b) $x_{2}(t)$ of SDE model (1.2) using the EM method with stepsize $\Delta=0.01$ and initial value $x_{0}=(0.7,0.15)^{T}$. Times series of the correlated Brownian motions $B_{2}(t)$ and $B_{4}(t)$ is shown in (c). 


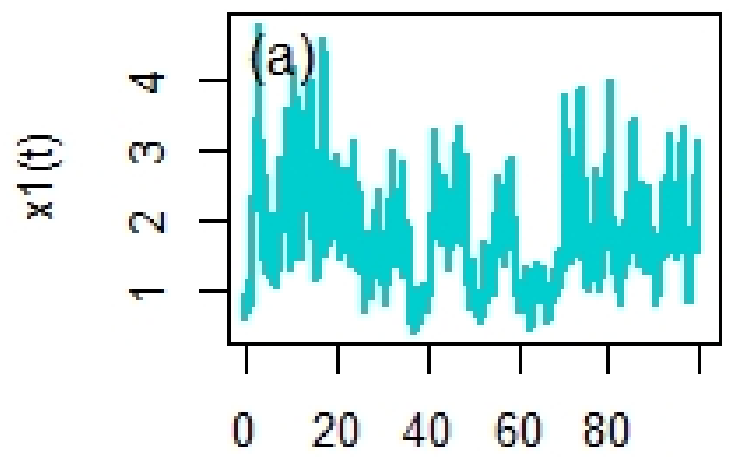

Time

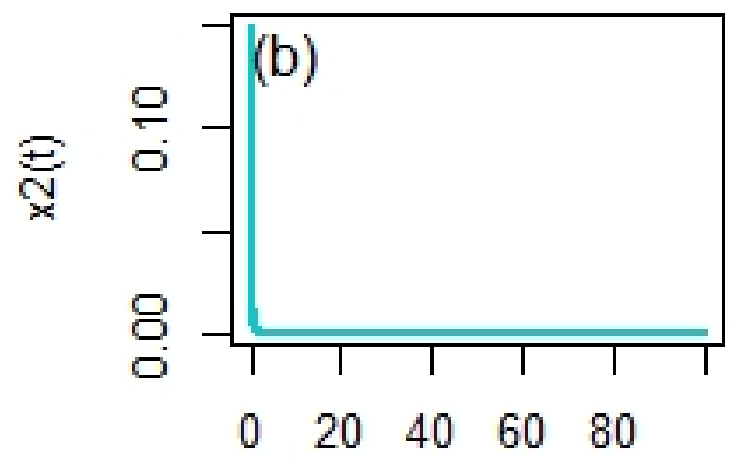

Time

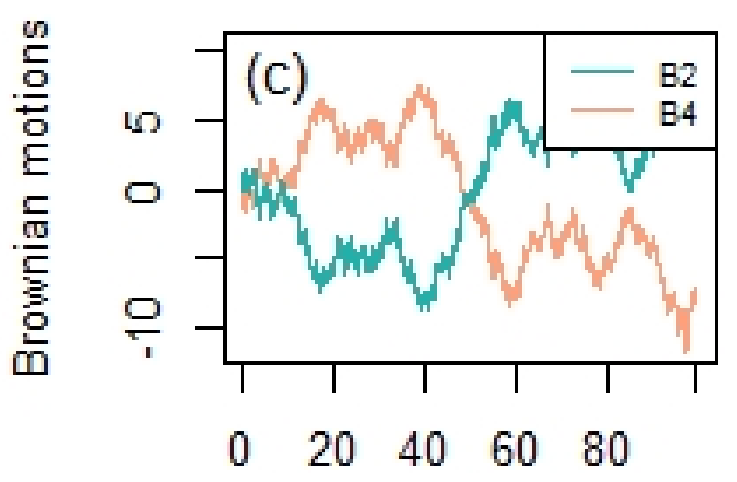

Time

Figure 3: With the system parameters given in Example 5.3, we obtain the computer simulations of the paths (a) $x_{1}(t)$ and (b) $x_{2}(t)$ of SDE model (1.2) using the EM method with stepsize $\Delta=0.01$ and initial value $x_{0}=(0.7,0.15)^{T}$. Times series of the correlated Brownian motions $B_{2}(t)$ and $B_{4}(t)$ is shown in $(\mathrm{c})$. 

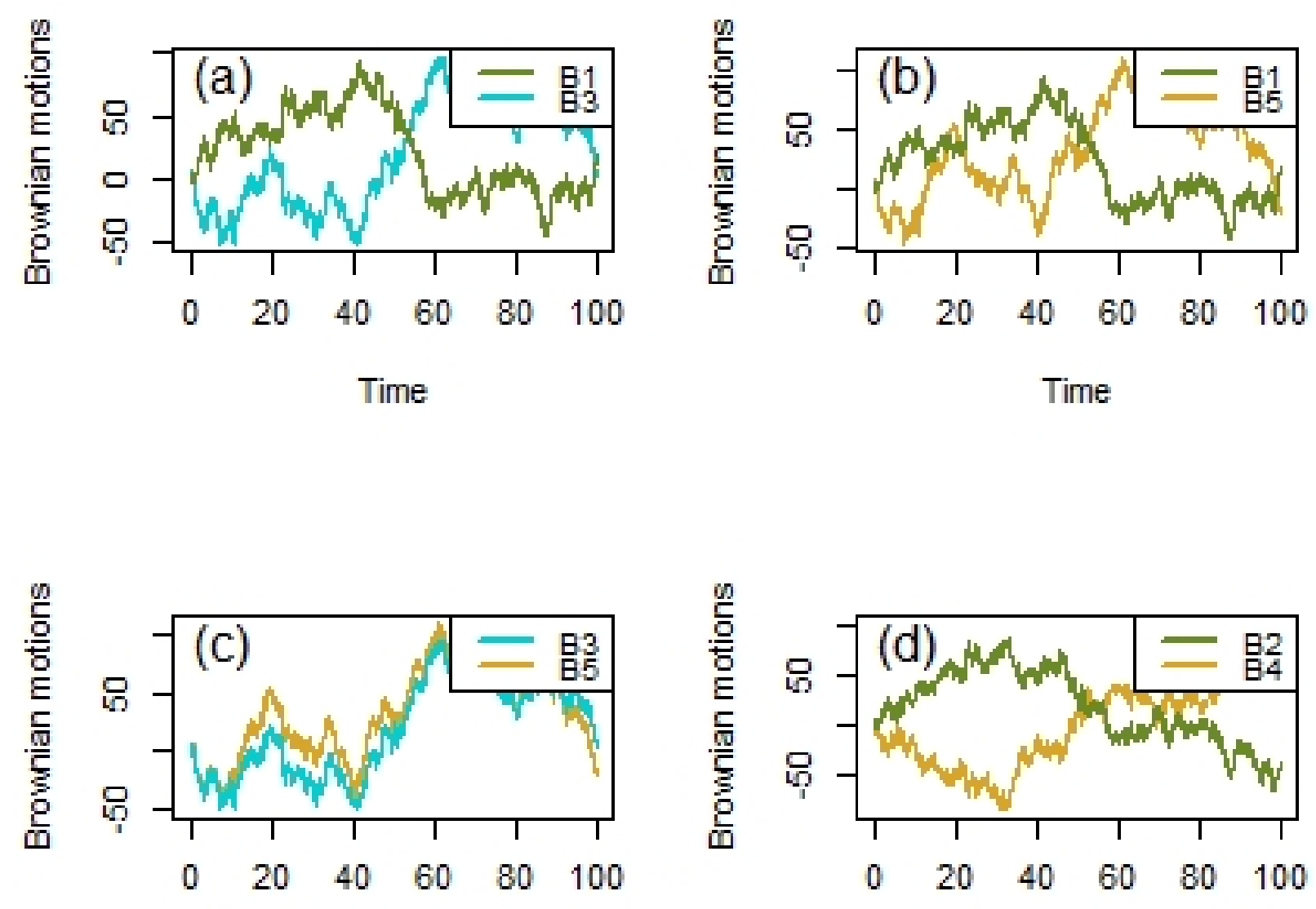

Time

Time
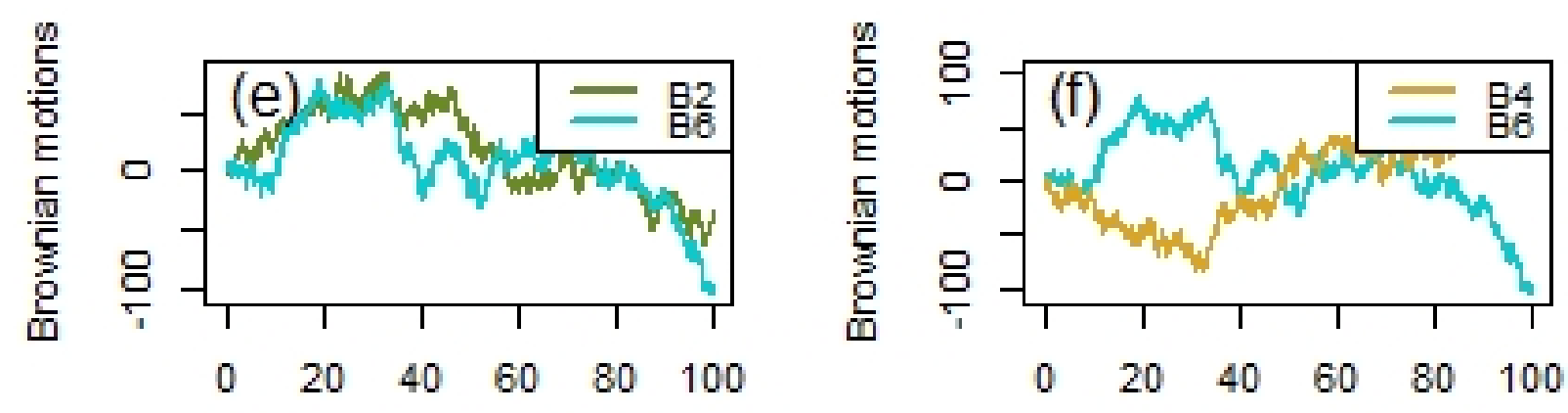

Time

Time

Figure 4: Time series of the correlated Brownian motions adopted in Example 5.4 and 5.5. 


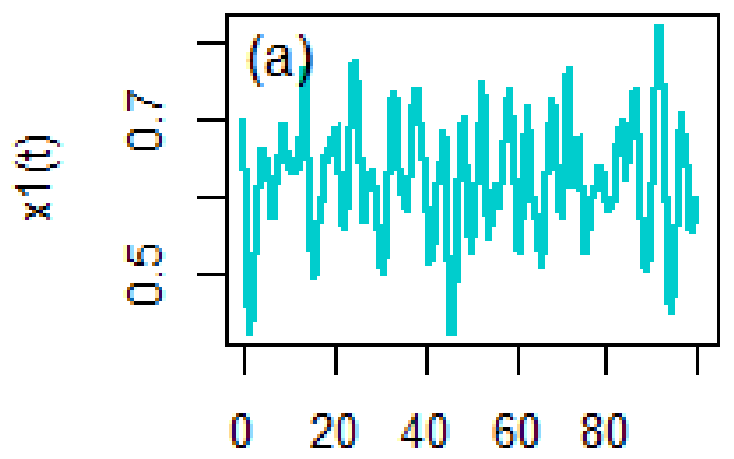

Time

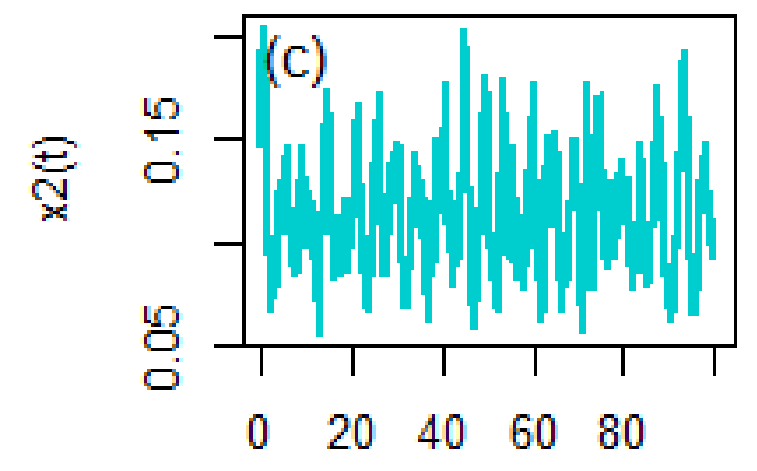

Time
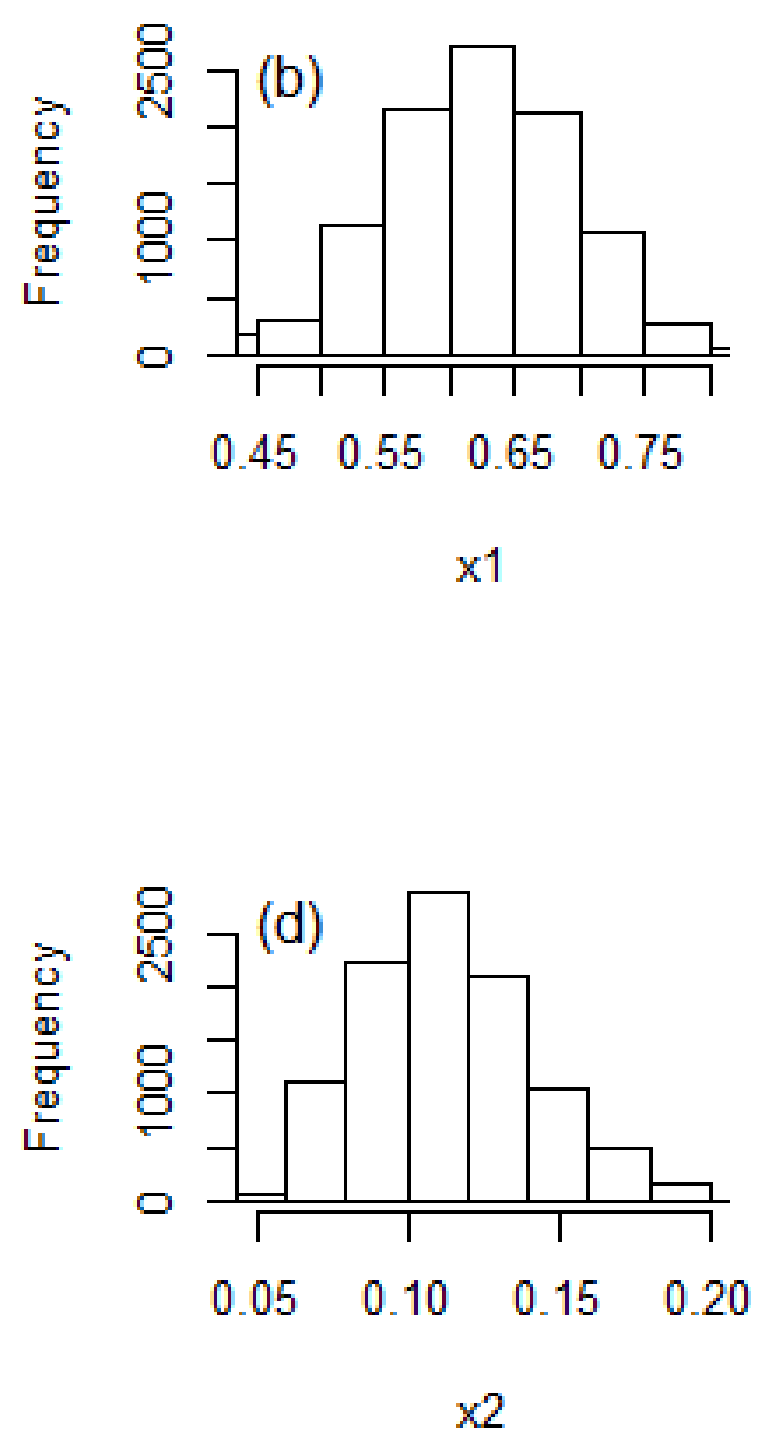

Figure 5: Numerical simulations of the paths (a) $x_{1}(t)$ and (c) $x_{2}(t)$ of SDE model (1.2) based on the model parameters described in Example 5.4 using the EM technique with stepsize $\Delta=0.01$ and initial value $x_{0}=(0.7,0.15)^{T}$, followed by the histograms for the SDE paths (b) $x_{1}(t)$ and (d) $x_{2}(t)$. 


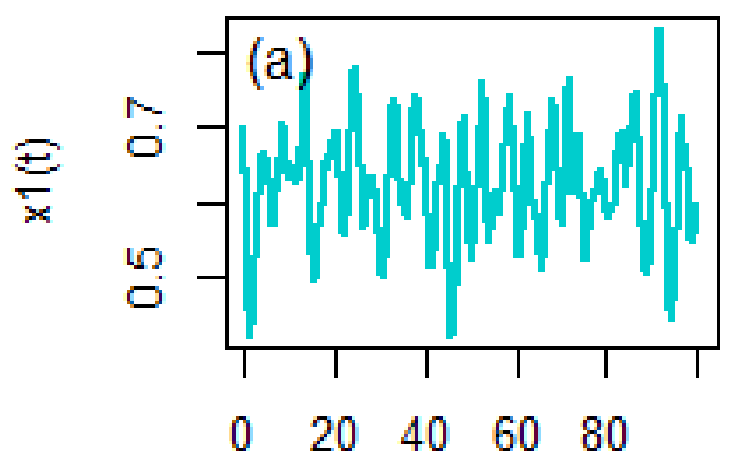

Time

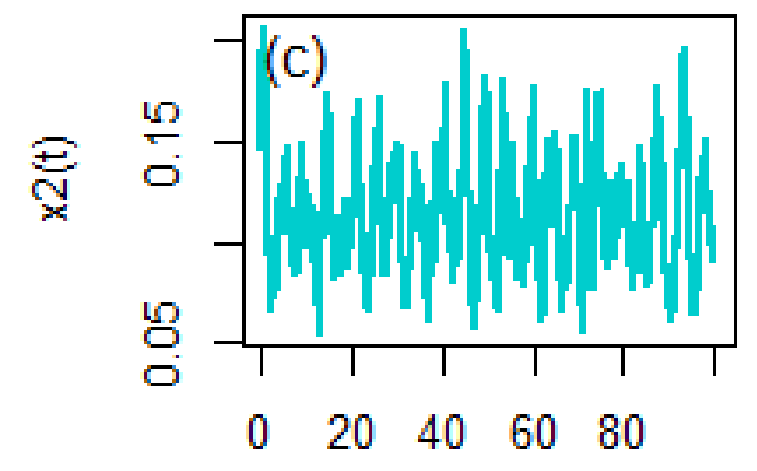

Time
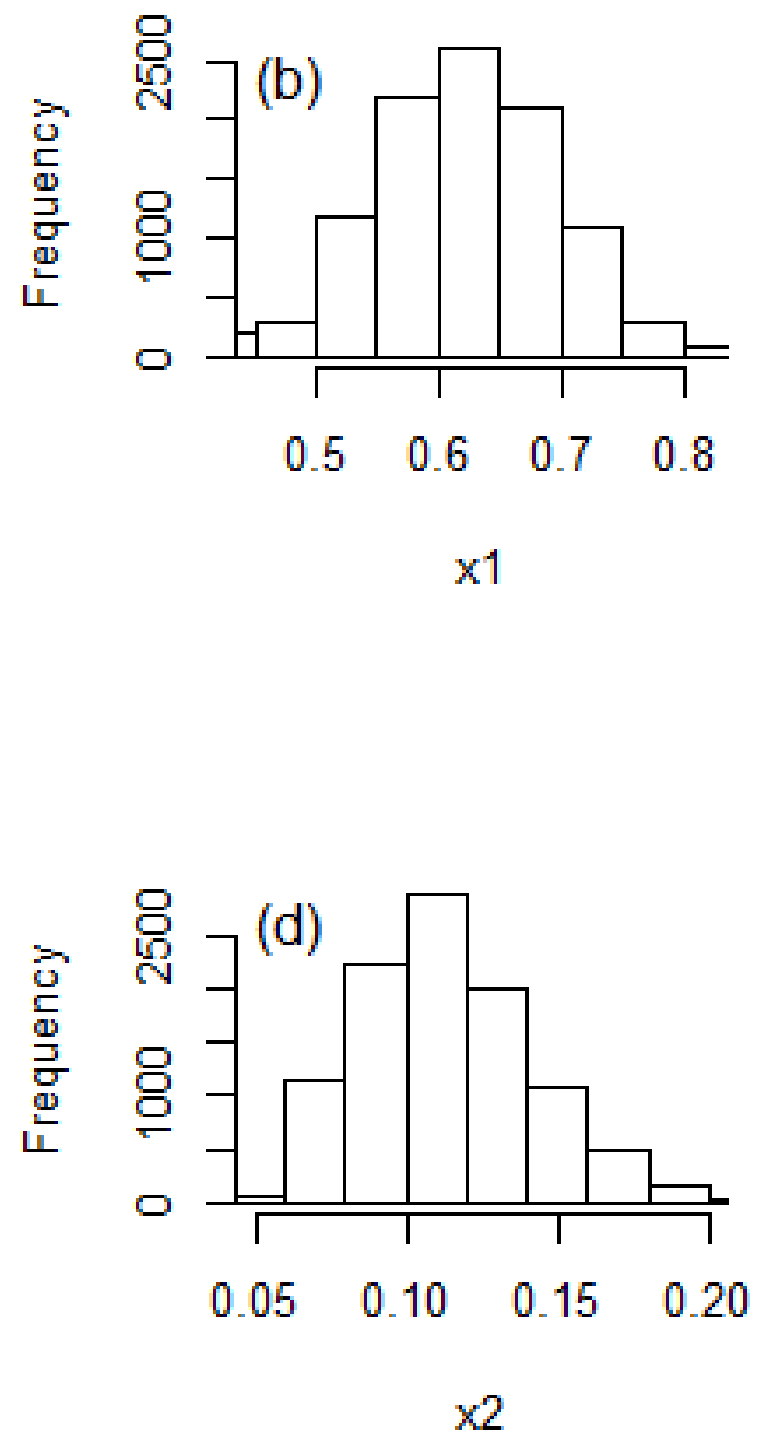

Figure 6: Computer simulations of the paths (a) $x_{1}(t)$ and (c) $x_{2}(t)$ of SDE model (1.3) based on the model parameters provided in Example 5.5 using the EM technique with stepsize $\Delta=0.01$ and initial value $x_{0}=(0.7,0.15)^{T}$, followed by the histograms for the SDE paths $(\mathrm{b}) x_{1}(t)$ and (d) $x_{2}(t)$. 
[4] C. S. Holling, "Some characteristics of simple types of predation and parasitism1," The Canadian Entomologist, vol. 91, no. 7, pp. 385-398, 1959.

[5] C. Jost, O. Arino, and R. Arditi, "About deterministic extinction in ratio-dependent predatorprey models," Bulletin of Mathematical Biology, vol. 61, no. 1, pp. 19-32, 1999.

[6] R. Arditi and L. R. Ginzburg, "Coupling in predator-prey dynamics: ratio-dependence," Journal of theoretical biology, vol. 139, no. 3, pp. 311-326, 1989.

[7] — How Species Interact: Altering the Standard View on Trophic Ecology. Oxford University Press, 2012, vol. NY,204pp.

[8] C. Walters, D. Pauly, V. Christensen, and J. F. Kitchell, "Representing density dependent consequences of life history strategies in aquatic ecosystems: Ecosim ii," Ecosystems, vol. 3, no. 1, pp. $70-83,2000$.

[9] R. N. Ahrens, C. J. Walters, and V. Christensen, "Foraging arena theory," Fish and Fisheries, vol. 13, no. 1, pp. 41-59, 2012.

[10] H. Li and Y. Takeuchi, "Dynamics of the density dependent predator-prey system with beddington-deangelis functional response," Journal of Mathematical Analysis and Applications, vol. 374, no. 2, pp. 644-654, 2011.

[11] B. Basener and D. S. Ross, "Booming and crashing populations and easter island," SIAM Journal on Applied Mathematics, vol. 65, no. 2, pp. 684-701, 2004.

[12] I. Mirzaev and D. M. Bortz, "Criteria for linearized stability for a size-structured population model," arXiv preprint arXiv:1502.02754, 2015.

[13] I. Stott, S. Townley, and D. J. Hodgson, "A framework for studying transient dynamics of population projection matrix models," Ecology Letters, vol. 14, no. 9, pp. 959-970, 2011.

[14] L. E. McMullen, P. De Leenheer, J. D. Tonkin, and D. A. Lytle, "High mortality and enhanced recovery: modelling the countervailing effects of disturbance on population dynamics," Ecology letters, vol. 20, no. 12, pp. 1566-1575, 2017.

[15] G. Guzzetta, F. Montarsi, F. A. Baldacchino, M. Metz, G. Capelli, A. Rizzoli, A. Pugliese, R. Rosà, P. Poletti, and S. Merler, "Potential risk of dengue and chikungunya outbreaks in northern italy based on a population model of aedes albopictus (diptera: Culicidae)," PLoS neglected tropical diseases, vol. 10, no. 6, p. e0004762, 2016.

[16] T. A. Morrison, A. B. Estes, S. A. Mduma, H. T. Maliti, H. Frederick, H. Kija, M. Mwita, A. Sinclair, and E. M. Kohi, "Informing aerial total counts with demographic models: population growth of serengeti elephants not explained purely by demography," Conservation Letters, vol. 11, no. 3, p. e12413, 2018.

[17] D. Lindenmayer, H. P. Possingham, R. Lacy, M. McCarthy, and M. Pope, "How accurate are population models? lessons from landscape-scale tests in a fragmented system," Ecology letters, vol. 6, no. 1, pp. 41-47, 2003.

[18] T. W. Green, D. H. Slone, E. D. Swain, M. S. Cherkiss, M. Lohmann, F. J. Mazzotti, and K. G. Rice, "Evaluating effects of everglades restoration on american crocodile populations in south florida using a spatially-explicit, stage-based population model," Wetlands, vol. 34, no. 1, pp. 213-224, 2014.

[19] D. T. Crouse, L. B. Crowder, and H. Caswell, "A stage-based population model for loggerhead sea turtles and implications for conservation," Ecology, vol. 68, no. 5, pp. 1412-1423, 1987. 
[20] R. Cook, "A rough guide to population change in exploited fish stocks," Ecology letters, vol. 3, no. 5, pp. 394-398, 2000.

[21] X. Mao, G. Marion, and E. Renshaw, "Environmental Brownian noise suppresses explosions in population dynamics," Stochastic Processes and their Applications, vol. 97, no. 1, pp. 95-110, 2002.

[22] X. Mao, C. Yuan, and J. Zou, "Stochastic differential delay equations of population dynamics," Journal of Mathematical Analysis and Applications, vol. 304, no. 1, pp. 296-320, 2005.

[23] X. Mao, "Stationary distribution of stochastic population systems," Systems $\&$ Control Letters, vol. 60, no. 6, pp. 398-405, 2011.

[24] X. Li, D. Jiang, and X. Mao, "Population dynamical behavior of lotka-volterra system under regime switching," Journal of Computational and Applied Mathematics, vol. 232, no. 2, pp. 427448, 2009.

[25] X. Li, A. Gray, D. Jiang, and X. Mao, "Sufficient and necessary conditions of stochastic permanence and extinction for stochastic logistic populations under regime switching," Journal of Mathematical Analysis and applications, vol. 376, no. 1, pp. 11-28, 2011.

[26] C. Ji, D. Jiang, and N. Shi, "Analysis of a predator-prey model with modified leslie-gower and holling-type ii schemes with stochastic perturbation," Journal of Mathematical Analysis and Applications, vol. 359, no. 2, pp. 482-498, 2009.

[27] J. Lv and K. Wang, "Asymptotic properties of a stochastic predator-prey system with holling ii functional response," Communications in Nonlinear Science and Numerical Simulation, vol. 16, no. 10, pp. 4037-4048, 2011.

[28] Q. Liu, L. Zu, and D. Jiang, "Dynamics of stochastic predator-prey models with holling ii functional response," Communications in Nonlinear Science and Numerical Simulation, vol. 37, pp. 62-76, 2016.

[29] X. Zhang, Y. Li, and D. Jiang, "Dynamics of a stochastic holling type ii predator-prey model with hyperbolic mortality," Nonlinear Dynamics, vol. 87, no. 3, pp. 2011-2020, 2017.

[30] R. He, Z. Xiong, D. Hong, and H. Yin, "Analysis of a stochastic ratio-dependent one-predator and two-mutualistic-preys model with markovian switching and holling type iii functional response," Advances in Difference Equations, vol. 2016, no. 1, p. 285, 2016.

[31] X. Mao and C. Yuan, Stochastic differential equations with Markovian switching. Imperial College Press, 2006.

[32] C. Ji and D. Jiang, "Dynamics of a stochastic density dependent predator-prey system with beddington-deangelis functional response," Journal of Mathematical Analysis and Applications, vol. 381, no. 1, pp. 441-453, 2011.

[33] B. E. Kendall, O. N. Bjørnstad, J. Bascompte, T. H. Keitt, and W. F. Fagan, "Dispersal, environmental correlation, and spatial synchrony in population dynamics," The American Naturalist, vol. 155 , no. 5, pp. 628-636, 2000.

[34] A. Liebhold, W. D. Koenig, and O. N. Bjørnstad, "Spatial synchrony in population dynamics," Annu. Rev. Ecol. Evol. Syst., vol. 35, pp. 467-490, 2004.

[35] A. Hening and D. H. Nguyen, "Stochastic lotka-volterra food chains," Journal of mathematical biology, pp. 1-29, 2017. 
[36] N. T. Dieu, D. H. Nguyen, N. H. Du, and G. Yin, "Classification of asymptotic behavior in a stochastic sir model," SIAM Journal on Applied Dynamical Systems, vol. 15, no. 2, pp. 1062-1084, 2016. [Online]. Available: https://doi.org/10.1137/15M1043315

[37] N. T. Dieu, N. H. Du, H. Nguyen, and G. Yin, "Protection zones for survival of species in random environment," SIAM Journal on Applied Mathematics, vol. 76, no. 4, pp. 1382-1402, 2016.

[38] R. Rudnicki, "Long-time behaviour of a stochastic prey-predator model," Stochastic Processes and their Applications, vol. 108, no. 1, pp. 93-107, 2003.

[39] A. Gray, D. Greenhalgh, L. Hu, X. Mao, and J. Pan, "A stochastic differential equation sis epidemic model," SIAM Journal on Applied Mathematics, vol. 71, no. 3, pp. 876-902, 2011.

[40] X. Mao, Stochastic differential equations and applications. Elsevier, 2007.

[41] R. Khasminskii, Stochastic stability of differential equations. Springer Science \& Business Media, 2011, vol. 66 .

[42] D. J. Higham, "An algorithmic introduction to numerical simulation of stochastic differential equations," SIAM review, vol. 43, no. 3, pp. 525-546, 2001.

[43] X. Mao, "Numerical solutions of stochastic differential delay equations under the generalized khasminskii-type conditions," Applied Mathematics and Computation, vol. 217, no. 12, pp. 5512$5524,2011$. 